\title{
Non-target Discovery of Per- and Polyfluoroalkyl Substances in Atmospheric Particulate Matter and Gaseous Phase Using Cryogenic Air Sampler
}

Nanyang $\mathrm{Yu}^{\dagger}$, Haozhe $\mathrm{Wen}^{\dagger}$, Xuebing Wang ${ }^{\dagger}$, Eriko Yamazaki ${ }^{\dagger}$, Sachi Taniyasu ${ }^{\dot{+}}$,

Nobuyoshi Yamashita ${ }^{\ddagger}$, Hongxia $\mathrm{Yu}^{\dagger}$ and Si Wei ${ }^{\dagger^{*}}$

†State Key Laboratory of Pollution Control and Resource Reuse, School of the Environment, Nanjing University, Nanjing, People's Republic of China

National Institute of Advanced Industrial Science and Technology (AIST), Tsukuba, Ibaraki, 305-8569, Japan

*Phone: +86 258968 0356; fax: +86 258968 0356; e-mail: weisi@nju.edu.cn (S.W.).

Number of pages: 31

Number of Tables: 7

Number of Figures: 12 


\section{Contents}

Section 1. Chemicals and Reagents

Page S3

Table S1. The compound name, abbreviations, molecular formula, supplier and purity of standards

Page S4

Section 2. Sample Preparation

Page S5

Table S2. The Specification, precondition and elution solution of

SPE cartridges

Page S6

Section 3. UPLC-HRMS Analysis

Page S7

Table S3. Mobile phase gradient elution condition

Page S8

Table S4. The procedural recovery $\left(20 \mu \mathrm{L} \mathrm{L}^{-1}\right)$ and the sensitivity of

UPLC-HRMS analysis with known PFASs standards

Page S9

Section 4. Non-target Screening and Identification of PFASs

Page S10

Table S5. The identified PFASs with level 4 or above

Page S12

Table S6. The CAS number, IUPAC name and SMILE for the identified

PFASs with level 1 and level 2

Page S15

Figure S1. Identification of PFOA (m/z 412.96643)

Page S17

Figure S2. Identification of PFOS (m/z 498.93021)

Page S18

Figure S3. Identification of 6:2 FTS (m/z 426.96791)

Page S19

Figure S4. Identification of 6:2 Cl-PFESA (m/z 530.89558)

Page S20

Figure S5. Identification of 6:2 diPAP (m/z 788.97505)

Page S21

Figure S6. Identification of H-PFCA

Page S22

Figure S7. Identification of G03 (m/z 532.92540) in Class 7

Page S23 
Figure S8. Extract ion chromatogram G01 (m/z 432.93179) in Class 7

Page S24

Figure S9. Identification of H04 (m/z 698.91074) in Class 8

Page S25

Figure S10. Identification of I02 (m/z 714.90565) in Class 9

Page S26

Figure S11. Identification of J02 (m/z 758.87102) in Class 10

Page S27

Figure S12. Isotope distribution of K02 (m/z 824.9036) in Class 11

Page S28

Table S7. Potential PFASs homologues found as Level 5

Page S29

References

Page S31 
Section 1. Chemicals and Reagents.

Formic acid (HPLC grade, purity: 99\%) and ammonium hydroxide solution $\sim 25 \%$ (HPLC grade) was purchased from ROE SCIENTIFIC ING(USA). Ammonium acetate (HPLC grade) was purchased from CNW technologies GmbH (Duesseldorf, Germany). Water (LCMS, purity> 99.99\%)was purchased from Fisher (USA). Methanol (HPLC grade, purity: 99.9\%) was purchased from Merck (Germany). The detail information of standards is shown in Table S1. 
Table S1. The compound name, abbreviations, molecular formula, supplier and purity of standards.

\begin{tabular}{|c|c|c|c|c|}
\hline Compound name & Abbreviation & Molecular formula & Supplier & Purity \\
\hline Perfluorobutane sulfonate & PFBS & C4HF9O3S & Woke & $98 \%$ \\
\hline Perfluorohexane sulfonate & PFHxS & C6HF13O3S & Sigma-Aldrich & $98 \%$ \\
\hline n-perfluoro-1-octanesulfonate & PFOS & C8HF17O3S & Wellington Laboratories & $98 \%$ \\
\hline Perfluoropentanoate & PFPeA & C5HF9O2 & Sigma-Aldrich & $97 \%$ \\
\hline Perfluorohexanoate & PFHxA & C6HF11O2 & Sigma-Aldrich & $97 \%$ \\
\hline Perfluoroheptanoate & PFHpA & $\mathrm{C} 7 \mathrm{HF} 13 \mathrm{O} 2$ & Sigma-Aldrich & $99 \%$ \\
\hline Perfluorooctanoate & PFOA & C8HF15O2 & Alfa Aesar & $95 \%$ \\
\hline Perfluorononanoate & PFNA & C9HF17O2 & Alfa Aesar & $97 \%$ \\
\hline Perfluorodecanoate & PFDA & C10HF19O2 & Sigma-Aldrich & $98 \%$ \\
\hline Perfluoroundecanoate & PFUnDA & $\mathrm{C} 11 \mathrm{HF} 21 \mathrm{O} 2$ & Sigma-Aldrich & $95 \%$ \\
\hline Perfluorododecanoate & PFDoDA & $\mathrm{C} 12 \mathrm{HF} 23 \mathrm{O} 2$ & Sigma-Aldrich & $95 \%$ \\
\hline Perfluorotridecanoate & PFTrDA & $\mathrm{C} 13 \mathrm{HF} 25 \mathrm{O} 2$ & Sigma-Aldrich & $97 \%$ \\
\hline Perfluorotetradecanoate & PFTeDA & $\mathrm{C} 14 \mathrm{HF} 27 \mathrm{O} 2$ & Sigma-Aldrich & $97 \%$ \\
\hline $\begin{array}{l}\text { 2-(6-Chloro-1,1,2,2,3,3,4,4,5,5,6,6-dodecafluorohexoxy)- } \\
\text { 1,1,2,2-tetrafluoroethanesulfonic acid }\end{array}$ & $6: 2$ Cl-PFESA & $\mathrm{C} 8 \mathrm{HClF} 16 \mathrm{O} 4 \mathrm{~S}$ & Wellington Laboratories & $98 \%$ \\
\hline 3,3,4,4,5,5,6,6,7,7,8,8,8-tidecafluorooctanesulphonic acid & $6: 2$ FTS & C8H5F13O3S & Wellington Laboratories & $98 \%$ \\
\hline 2-perfluorooctyl ethanoic acid & $8: 2$ FTA & $\mathrm{C} 10 \mathrm{H} 3 \mathrm{~F} 17 \mathrm{O} 2$ & Wellington Laboratories & $98 \%$ \\
\hline 2-perfluorodecyl ethanoic acid & 10:2 FTA & $\mathrm{C} 12 \mathrm{H} 3 \mathrm{~F} 21 \mathrm{O} 2$ & Wellington Laboratories & $98 \%$ \\
\hline 2,2,3,3,4,4,5,5,6,6,7,7-dodecafluoroheptanoic acid & 7-H-PFHpA & $\mathrm{C} 7 \mathrm{H} 2 \mathrm{~F} 12 \mathrm{O} 2$ & Fluorochem & $98 \%$ \\
\hline
\end{tabular}


Section 2. Sample Preparation.

Sample preparation of quartz fiber filter containing atmospheric particulate matter used our method previous study. ${ }^{1}$ Each filter sample was placed into a $15 \mathrm{~mL}$ polypropylene centrifuge tube separately. Each sample will be subjected to four cycles of 10 minutes ultrasonic extractions in a $40{ }^{\circ} \mathrm{C}$ water bath with $5 \mathrm{~mL}, 5 \mathrm{~mL}, 5 \mathrm{~mL}$ and $3 \mathrm{~mL}$ of methanol, respectively. The samples were centrifuged at $5000 \mathrm{rpm}$ for five minutes after each cycle of ultrasonic extraction and the four extracted supernatants for one sample were combined in a $15 \mathrm{~mL}$ polypropylene centrifuge tube.

Liquid samples collected by CMS were enriched through the sample preparation method in our previous study of water samples. ${ }^{2}$ Each sample was enriched by solid phase extraction (SPE) using Oasis MAX cartridge (6 cc, 500 mg, Waters, Ireland), Oasis MCX cartridge (6 cc, $500 \mathrm{mg}$, Waters, Ireland) and Oasis HLB cartridge (6 cc, $500 \mathrm{mg}$, Waters, Ireland) sequentially. Precondition and elution solution for each cartridge are listed in Table S2. The eluent is collected in a $15 \mathrm{~mL}$ polypropylene centrifuge tube for concentration under nitrogen and the eluents of the three cartridges for the same sample were combined in one $15 \mathrm{~mL}$ polypropylene centrifuge tube.

All the ultrasonic extract and SPE eluent were reduced to a volume of $1 \mathrm{~mL}$ by evaporation under a gentle stream of nitrogen. The final concentrated extract was passed through a polypropylene-membrane syringe filter (Acrodisc GHP, $13 \mathrm{~mm}, 0.2$ $\mu \mathrm{m}$, Waters) and transferred into a polypropylene vial for instrumental analysis. 
Table S2. The Specification, precondition and elution solution of SPE cartridges.

\begin{tabular}{|c|c|c|c|}
\hline SPE cartridge & Specification & Precondition solution & Elution solution \\
\hline Oasis MAX & $6 \mathrm{cc}, 500 \mathrm{mg}$ & $\begin{array}{c}10 \mathrm{~mL} 2 \% \mathrm{NH} 4 \mathrm{OH} \text { in methanol } \\
10 \mathrm{~mL} \text { methanol } \\
10 \mathrm{~mL} \text { water }\end{array}$ & $\begin{array}{l}10 \mathrm{~mL} 2 \% \\
\mathrm{HCOOH} \text { in } \\
\text { methanol }\end{array}$ \\
\hline Oasis MCX & $6 \mathrm{cc}, 500 \mathrm{mg}$ & $\begin{array}{c}10 \mathrm{~mL} 2 \% \mathrm{HCOOH} \text { in methanol } \\
10 \mathrm{~mL} \text { methanol } \\
10 \mathrm{~mL} \text { water }\end{array}$ & $\begin{array}{l}10 \mathrm{~mL} 2 \% \\
\mathrm{NH} 4 \mathrm{OH} \text { in } \\
\text { methanol }\end{array}$ \\
\hline Oasis HLB & $6 \mathrm{cc}, 500 \mathrm{mg}$ & $\begin{array}{c}10 \mathrm{~mL} \text { n-hexane } \\
\text { 10mL dichloromethane } \\
10 \mathrm{~mL} \text { methanol } \\
10 \mathrm{~mL} \text { water }\end{array}$ & $10 \mathrm{~mL}$ methanol \\
\hline
\end{tabular}


Section 3. UPLC-HRMS Analysis.

UPLC-HRMS Analysis for non-target screening was performed on ultra-performance liquid chromatography (UPLC; UltiMate 3000 Series, Thermo Fisher Scientific, Bremen, Germany) coupled with a high-resolution orbitrap mass spectrometer ( $Q$ Exactive Focus, Thermo Fisher Scientific, Bremen, Germany) with an electrospray ionization (ESI) source operating in negative ion mode. Sample separation used a C18 column (ACQUITY UPLC BEH C18, $1.7 \mu \mathrm{m}, 2.1 \times 150 \mathrm{~mm}$, Waters, Ireland) kept at 40 ${ }^{\circ} \mathrm{C}$. The mobile phases used were A: $2 \mathrm{mmol}$ ammonium acetate, $5 \%$ methanol in water and $\mathrm{B}$ : methanol with the flow rate of $0.3 \mathrm{~L} / \mathrm{min}$. Mobile phase gradient elution conditions are shown in Table S3. The injection volume was $10 \mu \mathrm{L}$. The parameters of the ion source were: spray voltage, $2500 \mathrm{~V}$; capillary temperature, $320{ }^{\circ} \mathrm{C}$; sheath gas, 48; aux gas, 11; spare gas, 2. The Q Exactive Focus was operated in Full MS combined with Discovery mode data dependent MS/MS (dd $\mathrm{MS}^{2}$ ). The resolution of Full MS is 70000 with the mass range of $\mathrm{m} / \mathrm{z} 80$ to 1000 and the resolution of dd $\mathrm{MS}^{2}$ is 17500 with fixed first mass of $50 \mathrm{~m} / \mathrm{z}$. The other parameters were: nce, 20, 35, 50; AGC target, 5e4; loop count, 3 . 
Table S3. Mobile phase gradient elution condition.

\begin{tabular}{ccc}
\hline Time $/$ min & $\begin{array}{c}\% \text { A } \\
\text { (2 mmol ammonium acetate } \\
5 \% \text { methanol in water) }\end{array}$ & $\begin{array}{c}\% \\
\text { (methanol) }\end{array}$ \\
\hline 0 & 100 & 0 \\
1 & 100 & 25 \\
9 & 75 & 50 \\
18 & 50 & 75 \\
28 & 25 & 100 \\
39 & 0 & 100 \\
48 & 0 & 0 \\
48.1 & 100 & 0 \\
55 & 100 & \\
\hline
\end{tabular}


Table S4. The procedural recovery $(20 \mu \mathrm{g} \mathrm{L}-1)$ and the sensitivity of UPLC-HRMS analysis with known PFASs standards.

\begin{tabular}{|c|c|c|c|c|c|c|c|c|c|c|c|}
\hline \multirow[b]{2}{*}{ Analyte } & \multirow{2}{*}{$\begin{array}{l}\text { Theoretical } \\
\text { mass / Da }\end{array}$} & \multicolumn{4}{|c|}{$1 \mu \mathrm{g} \mathrm{L} \mathrm{L}^{-1}$} & \multicolumn{4}{|c|}{$5 \mu g \mathrm{~L}^{-1}$} & \multicolumn{2}{|c|}{ Procedural recovery $(n=3) / \operatorname{Mean}($ S.D. $)$} \\
\hline & & $\begin{array}{l}\mathrm{RT} \\
/ \min \end{array}$ & $\begin{array}{c}\text { Experimental } \\
\text { mass / Da }\end{array}$ & $\begin{array}{l}\text { Error } \\
/ \mathrm{mDa}\end{array}$ & $\begin{array}{l}\text { Error } \\
/ \mathrm{ppm}\end{array}$ & $\begin{array}{l}\mathrm{RT} \\
/ \mathrm{min}\end{array}$ & $\begin{array}{c}\text { Experimental } \\
\text { mass / Da }\end{array}$ & $\begin{array}{l}\text { Error } \\
/ \mathrm{mDa}\end{array}$ & $\begin{array}{l}\text { Error } \\
\text { / ppm }\end{array}$ & $\begin{array}{c}\text { Particulate } \\
\text { Matter }\end{array}$ & $\begin{array}{c}\text { Gaseous } \\
\text { Phase }\end{array}$ \\
\hline PFBS & 298.94299 & 17.22 & 298.94312 & 0.13 & 0.43 & 17.12 & 298.94305 & 0.06 & 0.20 & $102.1 \%(0.03)$ & $116.4 \%(0.01)$ \\
\hline PFHxS & 398.93660 & 23.87 & 398.93661 & 0.01 & 0.03 & 23.78 & 398.93658 & -0.02 & -0.05 & $101.3 \%(0.07)$ & $90.4 \%(0.04)$ \\
\hline PFOS & 498.93022 & 27.83 & 498.93036 & 0.14 & 0.28 & 27.75 & 498.93030 & 0.08 & 0.16 & $103.1 \%(0.04)$ & $94.5 \%(0.20)$ \\
\hline PFPeA & 262.97601 & 15.47 & 262.97620 & 0.19 & 0.72 & 15.41 & 262.97614 & 0.13 & 0.49 & $104.3 \%(0.06)$ & $94.9 \%(0.02)$ \\
\hline PFHxA & 312.97281 & 20.29 & 312.97308 & 0.27 & 0.86 & 20.29 & 312.97296 & 0.15 & 0.48 & $85.6 \%(0.14)$ & $94.5 \%(0.06)$ \\
\hline PFHpA & 362.96962 & 23.49 & 362.96985 & 0.23 & 0.63 & 23.41 & 362.96991 & 0.29 & 0.80 & $101.9 \%(0.06)$ & $102.4 \%(0.06)$ \\
\hline PFOA & 412.96643 & 25.85 & 412.96671 & 0.28 & 0.68 & 25.78 & 412.96655 & 0.12 & 0.29 & $102.8 \%(0.05)$ & $100.2 \%(0.10)$ \\
\hline PFNA & 462.96323 & 27.73 & 462.96375 & 0.52 & 1.12 & 27.67 & 462.96338 & 0.15 & 0.32 & $102.7 \%(0.04)$ & $102.4 \%(0.07)$ \\
\hline PFDA & 512.96004 & 29.29 & 512.96002 & -0.02 & -0.04 & 29.24 & 512.95990 & -0.14 & -0.27 & $107.1 \%(0.03)$ & $100.1 \%(0.18)$ \\
\hline PFUnDA & 562.95685 & 30.58 & 562.95721 & 0.36 & 0.64 & 30.56 & 562.95709 & 0.24 & 0.43 & $110.1 \%(0.05)$ & $99.5 \%(0.29)$ \\
\hline PFDoDA & 612.95365 & 31.77 & 612.95428 & 0.63 & 1.03 & 31.72 & 612.95404 & 0.39 & 0.64 & $135.9 \%(0.09)$ & $80.1 \%(0.10)$ \\
\hline PFTrDA & 662.95046 & 32.78 & 662.95087 & 0.41 & 0.62 & 32.72 & 662.95099 & 0.53 & 0.80 & $139.7 \%(0.11)$ & $74.5 \%(0.04)$ \\
\hline PFTeDA & 712.94726 & 33.66 & 712.94812 & 0.86 & 1.21 & 33.61 & 712.94781 & 0.55 & 0.77 & $111.1 \%(0.36)$ & $49.8 \%(0.02)$ \\
\hline
\end{tabular}


Section 4. Non-target Screening and Identification of PFASs.

The peak picking process of raw data is implemented by Compound Discoverer 3.0 (Thermo Fisher Scientific). The workflow composed of Input Files, Select Spectra, Align Retention Time, Detect Compounds, Group Compounds and Assign Compound Annotations, successively. Detailed parameters are Mass Tolerance 5 ppm, Maximum Shift 2 min, Intensity Tolerance 30\%, S/N threshold 3, Min. Peak Intensity 10000, Max. Peak Width 0.5 min, Min. \# Scans per Peak 5, Min. \# Isotopes 2 and RT Tolerance 0.2 $\min$.

PFASs homologues were found from peaks picked above using MATLAB script for PFAS Homologue Analysis described in our previous studies. ${ }^{1}$ In this script, the input data was the peak list including exact mass and retention time, and the output result was the homologue candidate list. The mass difference of any two peaks was calculated during the running of script. The PFASs homologues were identified by the mass difference of $49.99681 \mathrm{Da}(\mathrm{CF} 2$, mass error $<5 \mathrm{ppm})$ among the exact mass of peaks.

The PFASs homologues were screened by database firstly for identification. The database includes the laboratory's existing standards, the PFASs we have identified in previous studies $^{1,2}$ and the PFASs with MS/MS spectrum information in the Norman database $^{3}$. The database screening was using TraceFinder 4.1 General Quan (Thermo Fisher Scientific). PFASs information data including name, parent ion and product ion was imported into TraceFinder as Compound Database by CSV format. A target 
screening method was used to screen the PFASs in created Compound Database with Mass Precision 5, S/N Ratio Threshold 3 and Mass Tolerance 5 ppm for Peaks, Intensity Threshold 1000 and Mass Tolerance 5 ppm for Fragment Ions.

For the PFASs homologues that not matched database, molecular formula and MS/MS fragments were calculated by Compound Discoverer 3.0 (function Predict Compositions) and Qual Browser (Thermo Fisher Scientific). The elements setting included C (3-50), H (0-50), F (0-50), O (0-8), N (0-4), P (0-4), S (0-4), Cl (0-2). The mass tolerance was $5 \mathrm{ppm}$ for parent ion and $5 \mathrm{mDa}$ for fragments.

With Sirius and CSI:FingerID, the relationship of fragmentation and the formula of fragments were predicted based on the MS/MS spectra, which could assist identification of structure. The confidence of the identification results is based on previous study. ${ }^{4}$ Briefly, Level 1: confirmed structure by reference standard; Level 2a: probable structure matching literature or library spectrum data; Level $2 \mathrm{~b}$ : probable structure with diagnostic evidence but no standard or literature information is available for confirmation; Level 3: tentative candidate(s) with evidence existing for possible structure(s), but insufficient information for one exact structure only; Level 4: unequivocal molecular formula and Level 5: exact mass. 
Table S5. The identified PFASs with level 4 or above. The numbers in the "Particulate Matter" and "Gaseous Phase" show the distribution ratio in each phase and were converted by chromatographic peak area.

\begin{tabular}{|c|c|c|c|c|c|c|c|c|c|c|c|c|}
\hline \multirow[b]{2}{*}{ Class } & \multirow{2}{*}{$\begin{array}{c}\text { Serial } \\
\text { Number }\end{array}$} & \multirow[b]{2}{*}{ Formula } & \multirow[b]{2}{*}{ Level } & \multirow{2}{*}{$\begin{array}{c}\text { Theoretical } \\
\text { Molecular } \\
\text { Weight }\end{array}$} & \multirow{2}{*}{$\begin{array}{l}\text { Mass } \\
\text { Error } \\
\text { /ppm }\end{array}$} & \multirow{2}{*}{$\begin{array}{l}\mathrm{RT} \\
/ \mathrm{min}\end{array}$} & \multirow{2}{*}{$\begin{array}{l}\text { RT delta } \\
\text { /min }\end{array}$} & \multicolumn{4}{|c|}{ Particulate Matter } & \multirow{2}{*}{$\begin{array}{c}\text { Gaseous } \\
\text { Phase }\end{array}$} \\
\hline & & & & & & & & $>10 \mu \mathrm{m}$ & $10-2.5 \mu \mathrm{m}$ & $2.5-1 \mu \mathrm{m}$ & $<1 \mu \mathrm{m}$ & \\
\hline Class 1 & $\mathrm{~A} 01$ & C4HF7O2 & 2 & 213.98648 & $0.30 \sim 2.42$ & 9.06 & & 0.40 & 0.31 & & 0.29 & \\
\hline \multirow[t]{7}{*}{ PFCAs } & $\mathrm{A} 02$ & C5HF9O2 & 1 & 263.98328 & $0.01 \sim 0.82$ & 15.08 & $0.09 \sim 0.27$ & & & & 0.48 & 0.52 \\
\hline & $\mathrm{A} 03$ & C6HF11O2 & 1 & 313.98009 & $0.38 \sim 2.53$ & 19.82 & $0.00 \sim 0.22$ & 0.06 & 0.08 & & 0.70 & 0.16 \\
\hline & A04 & $\mathrm{C} 7 \mathrm{HF} 13 \mathrm{O} 2$ & 1 & 363.97690 & $0.04 \sim 2.40$ & 22.88 & $0.09 \sim 0.15$ & 0.11 & 0.09 & 0.07 & 0.67 & 0.06 \\
\hline & A05 & $\mathrm{C} 8 \mathrm{HF} 15 \mathrm{O} 2$ & 1 & 413.97370 & $0.29 \sim 1.78$ & 25.31 & $0.02 \sim 0.15$ & 0.05 & 0.18 & 0.17 & 0.50 & 0.11 \\
\hline & A06 & C9HF17O2 & 1 & 463.97051 & $0.26 \sim 1.51$ & 27.22 & $0.00 \sim 0.05$ & 0.29 & 0.12 & 0.19 & 0.10 & 0.31 \\
\hline & A07 & C10HF19O2 & 1 & 513.96732 & $0.04 \sim 0.08$ & 28.83 & $0.03 \sim 0.06$ & 0.26 & & 0.23 & & 0.51 \\
\hline & A08 & $\mathrm{C} 12 \mathrm{HF} 23 \mathrm{O} 2$ & 1 & 613.96093 & $1.03 \sim 1.24$ & 31.35 & $0.03 \sim 0.06$ & 0.61 & & & & 0.39 \\
\hline Class 2 & B01 & $\mathrm{C} 6 \mathrm{HF} 13 \mathrm{O} 3 \mathrm{~S}$ & 1 & 399.94388 & $0.80 \sim 1.77$ & 23.28 & $0.11 \sim 0.19$ & 0.06 & 0.58 & 0.18 & 0.18 & \\
\hline PFSAs & B02 & C8HF17O3S & 1 & 499.93749 & $0.09 \sim 2.39$ & 27.30 & $0.08 \sim 0.20$ & & 0.33 & 0.67 & & \\
\hline $\begin{array}{c}\text { Class } 3 \\
\mathrm{n}: 2 \text { FTSs }\end{array}$ & $\mathrm{C} 01$ & C8H5F13O3S & 1 & 427.97518 & $0.05 \sim 1.35$ & 25.18 & $0.06 \sim 0.22$ & & 0.96 & 0.04 & & \\
\hline $\begin{array}{c}\text { Class } 4 \\
\text { Cl-PFESAs }\end{array}$ & D01 & $\mathrm{C} 8 \mathrm{HClF} 16 \mathrm{O} 4 \mathrm{~S}$ & 1 & 531.90286 & $0.8 \sim 1.29$ & 29.29 & $0.11 \sim 0.16$ & 0.03 & 0.41 & 0.42 & 0.14 & \\
\hline Class 5 & E01 & C16H9F26O4P & 2 & 789.98233 & 0.44 & 33.04 & & 1.00 & & & & \\
\hline diPAPs & E02 & C18H9F30O4P & 2 & 889.97594 & 4.35 & 34.37 & & 1.00 & & & & \\
\hline \multirow{3}{*}{$\begin{array}{c}\text { Class } 6 \\
\text { H-PFCAs }\end{array}$} & F01 & $\mathrm{C} 7 \mathrm{H} 2 \mathrm{~F} 12 \mathrm{O} 2$ & 3 & 345.98632 & $0.42 \sim 1.84$ & 18.60 & & 0.10 & 0.20 & 0.09 & 0.57 & 0.04 \\
\hline & F02 & $\mathrm{C} 8 \mathrm{H} 2 \mathrm{~F} 14 \mathrm{O} 2$ & 3 & 395.98312 & $0.18 \sim 1.83$ & 21.42 & & 0.13 & 0.32 & 0.08 & 0.45 & 0.02 \\
\hline & $\mathrm{F} 03$ & $\mathrm{C} 9 \mathrm{H} 2 \mathrm{~F} 16 \mathrm{O} 2$ & 3 & 445.97993 & $0.76 \sim 1.93$ & 23.72 & & 0.15 & 0.37 & 0.12 & 0.36 & 0.01 \\
\hline
\end{tabular}




\begin{tabular}{|c|c|c|c|c|c|c|c|c|c|c|c|c|}
\hline \multirow[b]{2}{*}{ Class } & \multirow{2}{*}{$\begin{array}{c}\text { Serial } \\
\text { Number }\end{array}$} & \multirow[b]{2}{*}{ Formula } & \multirow[b]{2}{*}{ Level } & \multirow{2}{*}{$\begin{array}{c}\text { Theoretical } \\
\text { Molecular } \\
\text { Weight }\end{array}$} & \multirow{2}{*}{$\begin{array}{l}\text { Mass } \\
\text { Error } \\
\text { /ppm }\end{array}$} & \multirow{2}{*}{$\begin{array}{c}\mathrm{RT} \\
/ \mathrm{min}\end{array}$} & \multirow{2}{*}{$\begin{array}{l}\text { RT delta } \\
\text { /min }\end{array}$} & \multicolumn{4}{|c|}{ Particulate Matter } & \multirow{2}{*}{$\begin{array}{c}\text { Gaseous } \\
\text { Phase }\end{array}$} \\
\hline & & & & & & & & $>10 \mu \mathrm{m}$ & $10-2.5 \mu \mathrm{m}$ & $2.5-1 \mu \mathrm{m}$ & $<1 \mu \mathrm{m}$ & \\
\hline & F04 & $\mathrm{C} 10 \mathrm{H} 2 \mathrm{~F} 18 \mathrm{O} 2$ & 3 & 495.97674 & $0.22 \sim 1.83$ & 25.65 & & & 0.75 & & 0.25 & \\
\hline & F05 & $\mathrm{C} 11 \mathrm{H} 2 \mathrm{~F} 20 \mathrm{O} 2$ & 3 & 545.97354 & $0.51 \sim 1.88$ & 27.30 & & 0.06 & 0.53 & 0.17 & 0.24 & \\
\hline & F06 & $\mathrm{C} 12 \mathrm{H} 2 \mathrm{~F} 22 \mathrm{O} 2$ & 3 & 595.97035 & $0.52 \sim 1.75$ & 28.72 & & 0.11 & 0.41 & 0.23 & 0.25 & 0.0003 \\
\hline & F07 & $\mathrm{C} 13 \mathrm{H} 2 \mathrm{~F} 24 \mathrm{O} 2$ & 3 & 645.96716 & $0.79 \sim 2.49$ & 29.93 & & 0.09 & 0.45 & 0.26 & 0.20 & \\
\hline & F08 & $\mathrm{C} 14 \mathrm{H} 2 \mathrm{~F} 26 \mathrm{O} 2$ & 3 & 695.96396 & $0.46 \sim 1.91$ & 30.98 & & 0.08 & 0.43 & 0.30 & 0.19 & \\
\hline & F09 & $\mathrm{C} 15 \mathrm{H} 2 \mathrm{~F} 28 \mathrm{O} 2$ & 3 & 745.96077 & $0.98 \sim 1.80$ & 31.92 & & 0.10 & 0.46 & 0.29 & 0.14 & 0.0004 \\
\hline & F10 & $\mathrm{C} 16 \mathrm{H} 2 \mathrm{~F} 30 \mathrm{O} 2$ & 3 & 795.95758 & $0.79 \sim 2.74$ & 32.76 & & 0.09 & 0.48 & 0.33 & 0.10 & \\
\hline & F11 & $\mathrm{C} 17 \mathrm{H} 2 \mathrm{~F} 32 \mathrm{O} 2$ & 3 & 845.95438 & $0.09 \sim 3.20$ & 33.51 & & 0.11 & 0.43 & 0.33 & 0.13 & 0.002 \\
\hline & F12 & $\mathrm{C} 19 \mathrm{H} 2 \mathrm{~F} 36 \mathrm{O} 2$ & 3 & 945.94799 & $0.79 \sim 1.90$ & 34.81 & & 0.10 & 0.46 & 0.33 & 0.11 & \\
\hline Class 7 & G01 & $\mathrm{C} 7 \mathrm{HClF} 14 \mathrm{O} 3$ & 3 & 433.93907 & $0.00 \sim 1.56$ & 28.31 & & 0.42 & 0.45 & & & 0.13 \\
\hline \multirow[t]{4}{*}{ Cl-PFDEA } & G02 & C8HClF16O3 & 3 & 483.93587 & $0.23 \sim 1.51$ & 29.49 & & 0.24 & 0.44 & 0.03 & & 0.29 \\
\hline & G03 & C9HClF18O3 & 3 & 533.93268 & $0.04 \sim 0.15$ & 30.51 & & 0.29 & 0.25 & 0.22 & 0.24 & \\
\hline & G04 & $\mathrm{C} 10 \mathrm{HClF} 20 \mathrm{O} 3$ & 3 & 583.92949 & $0.26 \sim 2.35$ & 31.18 & & 0.21 & & & & 0.79 \\
\hline & G05 & $\mathrm{C} 11 \mathrm{HClF} 22 \mathrm{O} 3$ & 3 & 633.92629 & $0.32 \sim 0.36$ & 32.02 & & 0.50 & 0.50 & & & \\
\hline Class 8 & H01 & C9HClF18O4 & 3 & 549.92759 & $0.05 \sim 2.07$ & 30.72 & & & 0.31 & & & 0.69 \\
\hline \multirow[t]{3}{*}{ Cl-PFTrEA } & $\mathrm{H} 02$ & $\mathrm{C} 10 \mathrm{HClF} 20 \mathrm{O} 4$ & 3 & 599.92440 & $0.02 \sim 0.90$ & 31.46 & & & & & 0.26 & 0.74 \\
\hline & $\mathrm{H} 03$ & $\mathrm{C} 11 \mathrm{HClF} 22 \mathrm{O} 4$ & 3 & 649.92121 & $0.02 \sim 0.59$ & 32.22 & & 0.52 & 0.18 & 0.13 & 0.16 & \\
\hline & H04 & $\mathrm{C} 12 \mathrm{HClF} 24 \mathrm{O} 4$ & 3 & 699.91801 & $0.14 \sim 0.49$ & 32.95 & & 0.29 & 0.23 & 0.26 & 0.21 & \\
\hline Class 9 & $\mathrm{I} 01$ & $\mathrm{C} 11 \mathrm{HClF} 22 \mathrm{O} 5$ & 3 & 665.91612 & $0.23 \sim 0.32$ & 32.46 & & 0.14 & 0.15 & 0.14 & 0.15 & 0.42 \\
\hline Cl-PFTeEA & I02 & $\mathrm{C} 12 \mathrm{HClF} 24 \mathrm{O} 5$ & 3 & 715.91293 & $0.15 \sim 2.63$ & 33.11 & & 0.28 & 0.34 & 0.25 & 0.13 & \\
\hline Class 10 & J01 & $\mathrm{C} 12 \mathrm{HCl} 2 \mathrm{~F} 21 \mathrm{O} 6$ & 3 & 709.88149 & $0.25 \sim 1.28$ & 31.72 & & 0.80 & & & 0.20 & \\
\hline 2Cl-PFTeECA & J02 & $\mathrm{C} 13 \mathrm{HCl} 2 \mathrm{~F} 23 \mathrm{O} 6$ & 3 & 759.87829 & $0.26 \sim 0.42$ & 32.49 & & 0.31 & 0.23 & 0.24 & 0.21 & \\
\hline
\end{tabular}




\begin{tabular}{|c|c|c|c|c|c|c|c|c|c|c|c|c|}
\hline \multirow[b]{2}{*}{ Class } & \multirow{2}{*}{$\begin{array}{c}\text { Serial } \\
\text { Number }\end{array}$} & \multirow[b]{2}{*}{ Formula } & \multirow[b]{2}{*}{ Level } & \multirow{2}{*}{$\begin{array}{c}\text { Theoretical } \\
\text { Molecular } \\
\text { Weight }\end{array}$} & \multirow{2}{*}{$\begin{array}{l}\text { Mass } \\
\text { Error } \\
\text { /ppm }\end{array}$} & \multirow{2}{*}{$\begin{array}{l}\mathrm{RT} \\
/ \mathrm{min}\end{array}$} & \multirow{2}{*}{$\begin{array}{l}\text { RT delta } \\
\text { /min }\end{array}$} & \multicolumn{4}{|c|}{ Particulate Matter } & \multirow{2}{*}{$\begin{array}{c}\text { Gaseous } \\
\text { Phase }\end{array}$} \\
\hline & & & & & & & & $>10 \mu \mathrm{m}$ & $10-2.5 \mu \mathrm{m}$ & $2.5-1 \mu \mathrm{m}$ & $<1 \mu \mathrm{m}$ & \\
\hline Class 11 & K01 & $\mathrm{C} 13 \mathrm{H} 2 \mathrm{ClF} 23 \mathrm{O} 6$ & 4 & 725.91727 & $0.11 \sim 1.28$ & 30.51 & & 0.55 & 0.45 & & & \\
\hline H,Cl-PFTeECA & K02 & $\mathrm{C} 16 \mathrm{H} 2 \mathrm{ClF} 29 \mathrm{O} 6$ & 4 & 875.90768 & $0.26 \sim 0.42$ & 32.98 & & 0.24 & 0.20 & 0.42 & 0.14 & \\
\hline Class 12 & L01 & C5H3F7O2 & 4 & 228.00213 & $1.24 \sim 2.86$ & 12.00 & & 0.002 & 0.03 & 0.43 & 0.53 & \\
\hline \multirow{3}{*}{$\mathrm{n}: 2$ FTAs } & L02 & $\mathrm{C} 8 \mathrm{H} 3 \mathrm{~F} 13 \mathrm{O} 2$ & 4 & 377.99255 & $0.00 \sim 1.59$ & 23.50 & & & & & & 1.00 \\
\hline & L03 & $\mathrm{C} 10 \mathrm{H} 3 \mathrm{~F} 17 \mathrm{O} 2$ & 1 & 477.98616 & $1.34 \sim 4.15$ & 23.77 & $0.28 \sim 0.34$ & 0.10 & 0.28 & 0.06 & 0.48 & 0.07 \\
\hline & L04 & $\mathrm{C} 12 \mathrm{H} 3 \mathrm{~F} 21 \mathrm{O} 2$ & 1 & 577.97977 & 0.14 & 27.91 & 0.16 & & 1.00 & & & \\
\hline Class 13 & M01 & C4HF7O3 & 4 & 229.98139 & 4.89 & 6.82 & & & 1.00 & & & \\
\hline \multirow[t]{2}{*}{ 1:n PFECAs } & M02 & C5HF9O3 & 4 & 279.97820 & $0.10 \sim 1.22$ & 11.83 & & & & & & 1.00 \\
\hline & M03 & C15HF29O3 & 4 & 779.94626 & 0.92 & 29.01 & & 1.00 & & & & \\
\hline
\end{tabular}


Table S6. The CAS number, IUPAC name and SMILE for the identified PFASs with level 1 and level 2.

\begin{tabular}{|c|c|c|c|c|c|}
\hline Class & Formula & Level & $\begin{array}{c}\text { CAS } \\
\text { Number }\end{array}$ & IUPAC Name & SMILE \\
\hline Class 1 & $\mathrm{C} 4 \mathrm{HF} 7 \mathrm{O} 2$ & 2 & $375-22-4$ & 2,2,3,3,4,4,4-heptafluorobutanoate & $\mathrm{C}(=\mathrm{O})(\mathrm{C}(\mathrm{C}(\mathrm{C}(\mathrm{F})(\mathrm{F}) \mathrm{F})(\mathrm{F}) \mathrm{F})(\mathrm{F}) \mathrm{F}) \mathrm{O}$ \\
\hline \multirow[t]{11}{*}{ PFCAs } & C5HF9O2 & 1 & $2706-90-3$ & $2,2,3,3,4,4,5,5,5$-nonafluoropentanoate & $\mathrm{C}(=\mathrm{O})(\mathrm{C}(\mathrm{C}(\mathrm{C}(\mathrm{C}(\mathrm{F})(\mathrm{F}) \mathrm{F})(\mathrm{F}) \mathrm{F})(\mathrm{F}) \mathrm{F})(\mathrm{F}) \mathrm{F}) \mathrm{O}$ \\
\hline & C6HF11O2 & 1 & $307-24-4$ & $2,2,3,3,4,4,5,5,6,6,6$-undecafluorohexanoic acid & $\mathrm{C}(=\mathrm{O})(\mathrm{C}(\mathrm{C}(\mathrm{C}(\mathrm{C}(\mathrm{C}(\mathrm{F})(\mathrm{F}) \mathrm{F})(\mathrm{F}) \mathrm{F})(\mathrm{F}) \mathrm{F})(\mathrm{F}) \mathrm{F})(\mathrm{F}) \mathrm{F}) \mathrm{O}$ \\
\hline & $\mathrm{C} 7 \mathrm{HF} 13 \mathrm{O} 2$ & 1 & $375-85-9$ & $2,2,3,3,4,4,5,5,6,6,7,7,7$-tridecafluoroheptanoate & $\mathrm{C}(=\mathrm{O})(\mathrm{C}(\mathrm{C}(\mathrm{C}(\mathrm{C}(\mathrm{C}(\mathrm{C}(\mathrm{F})(\mathrm{F}) \mathrm{F})(\mathrm{F}) \mathrm{F})(\mathrm{F}) \mathrm{F})(\mathrm{F}) \mathrm{F})(\mathrm{F}) \mathrm{F})(\mathrm{F}) \mathrm{F}) \mathrm{O}$ \\
\hline & C8HF15O2 & 1 & $335-67-1$ & $2,2,3,3,4,4,5,5,6,6,7,7,8,8,8-$ & $\mathrm{C}(=\mathrm{O})(\mathrm{C}(\mathrm{C}(\mathrm{C}(\mathrm{C}(\mathrm{C}(\mathrm{C}(\mathrm{C}(\mathrm{F})(\mathrm{F}) \mathrm{F})(\mathrm{F}) \mathrm{F})(\mathrm{F}) \mathrm{F})(\mathrm{F}) \mathrm{F})(\mathrm{F}) \mathrm{F})(\mathrm{F}) \mathrm{F})($ \\
\hline & & & & pentadecafluorooctanoic acid & F)F)O \\
\hline & C9HF17O2 & 1 & $375-95-1$ & $2,2,3,3,4,4,5,5,6,6,7,7,8,8,9,9,9-$ & $\mathrm{C}(=\mathrm{O})(\mathrm{C}(\mathrm{C}(\mathrm{C}(\mathrm{C}(\mathrm{C}(\mathrm{C}(\mathrm{C}(\mathrm{C}(\mathrm{F})(\mathrm{F}) \mathrm{F})(\mathrm{F}) \mathrm{F})(\mathrm{F}) \mathrm{F})(\mathrm{F}) \mathrm{F})(\mathrm{F}) \mathrm{F})(\mathrm{F})$ \\
\hline & & & & heptadecafluorononanoic acid & $\mathrm{F})(\mathrm{F}) \mathrm{F})(\mathrm{F}) \mathrm{F}) \mathrm{O}$ \\
\hline & $\mathrm{C} 10 \mathrm{HF} 19 \mathrm{O} 2$ & 1 & $335-76-2$ & $2,2,3,3,4,4,5,5,6,6,7,7,8,8,9,9,10,10,10-$ & $\mathrm{C}(=\mathrm{O})(\mathrm{C}(\mathrm{C}(\mathrm{C}(\mathrm{C}(\mathrm{C}(\mathrm{C}(\mathrm{C}(\mathrm{C}(\mathrm{C}(\mathrm{F})(\mathrm{F}) \mathrm{F})(\mathrm{F}) \mathrm{F})(\mathrm{F}) \mathrm{F})(\mathrm{F}) \mathrm{F})(\mathrm{F}) \mathrm{F})($ \\
\hline & & & & nonadecafluorodecanoic acid & $\mathrm{F}) \mathrm{F})(\mathrm{F}) \mathrm{F})(\mathrm{F}) \mathrm{F})(\mathrm{F}) \mathrm{F}) \mathrm{O}$ \\
\hline & $\mathrm{C} 12 \mathrm{HF} 23 \mathrm{O} 2$ & 1 & $307-55-1$ & $2,2,3,3,4,4,5,5,6,6,7,7,8,8,9,9,10,10,11,11,12,12,1$ & $\mathrm{C}(=\mathrm{O})(\mathrm{C}(\mathrm{C}(\mathrm{C}(\mathrm{C}(\mathrm{C}(\mathrm{C}(\mathrm{C}(\mathrm{C}(\mathrm{C}(\mathrm{C}(\mathrm{C}(\mathrm{F})(\mathrm{F}) \mathrm{F})(\mathrm{F}) \mathrm{F})(\mathrm{F}) \mathrm{F})(\mathrm{F}) \mathrm{F})($ \\
\hline & & & & 2-tricosafluorododecanoic acid & $\mathrm{F}) \mathrm{F})(\mathrm{F}) \mathrm{F})(\mathrm{F}) \mathrm{F})(\mathrm{F}) \mathrm{F})(\mathrm{F}) \mathrm{F})(\mathrm{F}) \mathrm{F})(\mathrm{F}) \mathrm{F}) \mathrm{O}$ \\
\hline \multirow{4}{*}{$\begin{array}{l}\text { Class } 2 \\
\text { PFSAs }\end{array}$} & C6HF13O3S & 1 & $355-46-4$ & $1,1,2,2,3,3,4,4,5,5,6,6,6$-tridecafluorohexane-1- & $\mathrm{C}(\mathrm{C}(\mathrm{C}(\mathrm{C}(\mathrm{F})(\mathrm{F}) \mathrm{S}(=\mathrm{O})(=\mathrm{O}) \mathrm{O})(\mathrm{F}) \mathrm{F})(\mathrm{F}) \mathrm{F})(\mathrm{C}(\mathrm{C}(\mathrm{F})(\mathrm{F}) \mathrm{F})(\mathrm{F}) \mathrm{F})($ \\
\hline & & & & sulfonic acid & F)F \\
\hline & C8HF17O3S & 1 & $1763-23-1$ & $1,1,2,2,3,3,4,4,5,5,6,6,7,7,8,8,8-$ & $\mathrm{C}(\mathrm{C}(\mathrm{C}(\mathrm{C}(\mathrm{C}(\mathrm{F})(\mathrm{F}) \mathrm{S}(=\mathrm{O})(=\mathrm{O}) \mathrm{O})(\mathrm{F}) \mathrm{F})(\mathrm{F}) \mathrm{F})(\mathrm{F}) \mathrm{F})(\mathrm{C}(\mathrm{C}(\mathrm{C}(\mathrm{F})($ \\
\hline & & & & heptadecafluorooctane-1-sulfonic acid & $\mathrm{F}) \mathrm{F})(\mathrm{F}) \mathrm{F})(\mathrm{F}) \mathrm{F})(\mathrm{F}) \mathrm{F}$ \\
\hline Class 3 & C8H5F13O3S & 1 & 27619-97-2 & $3,3,4,4,5,5,6,6,7,7,8,8,8$-Tridecafluorooctane-1- & $\mathrm{OS}(=\mathrm{O})(=\mathrm{O}) \mathrm{CCC}(\mathrm{F})(\mathrm{F}) \mathrm{C}(\mathrm{F})(\mathrm{F}) \mathrm{C}(\mathrm{F})(\mathrm{F}) \mathrm{C}(\mathrm{F})(\mathrm{F}) \mathrm{C}(\mathrm{F})(\mathrm{F}) \mathrm{C}(\mathrm{F}$ \\
\hline $\mathrm{n}: 2$ FTSs & & & & sulfonic acid & )$(\mathrm{F}) \mathrm{F}$ \\
\hline Class 4 & C8HClF16O4S & 1 & $756426-58-1$ & 2-(6-chloro-1,1,2,2,3,3,4,4,5,5,6,6-dodecafluoro- & $\mathrm{C}(\mathrm{C}(\mathrm{C}(\mathrm{C}(\mathrm{F})(\mathrm{F}) \mathrm{Cl})(\mathrm{F}) \mathrm{F})(\mathrm{F}) \mathrm{F})(\mathrm{C}(\mathrm{C}(\mathrm{OC}(\mathrm{C}(\mathrm{F})(\mathrm{F}) \mathrm{S}(=\mathrm{O})(=\mathrm{O})$ \\
\hline Cl-PFESAs & & & & hexoxy)-1,1,2,2-tetrafluoro-ethanesulfonic acid & $\mathrm{O})(\mathrm{F}) \mathrm{F})(\mathrm{F}) \mathrm{F})(\mathrm{F}) \mathrm{F})(\mathrm{F}) \mathrm{F}$ \\
\hline
\end{tabular}




\begin{tabular}{|c|c|c|c|c|c|}
\hline Class & Formula & Level & $\begin{array}{c}\text { CAS } \\
\text { Number }\end{array}$ & IUPAC Name & SMILE \\
\hline \multirow{5}{*}{$\begin{array}{l}\text { Class } 5 \\
\text { diPAPs }\end{array}$} & C16H9F26O4P & 2 & $57677-95-9$ & $\operatorname{bis}(3,3,4,4,5,5,6,6,7,7,8,8,8$-tridecafluorooctyl $)$ & $\mathrm{C}(\mathrm{COP}(=\mathrm{O})(\mathrm{O}) \mathrm{OCCC}(\mathrm{C}(\mathrm{C}(\mathrm{C}(\mathrm{C}(\mathrm{C}(\mathrm{F})(\mathrm{F}) \mathrm{F})(\mathrm{F}) \mathrm{F})(\mathrm{F}) \mathrm{F})(\mathrm{F}) \mathrm{F})$ \\
\hline & & & & hydrogen phosphate & $(\mathrm{F}) \mathrm{F})(\mathrm{F}) \mathrm{F}) \mathrm{C}(\mathrm{C}(\mathrm{C}(\mathrm{C}(\mathrm{C}(\mathrm{C}(\mathrm{F})(\mathrm{F}) \mathrm{F})(\mathrm{F}) \mathrm{F})(\mathrm{F}) \mathrm{F})(\mathrm{F}) \mathrm{F})(\mathrm{F}) \mathrm{F})(\mathrm{F}) \mathrm{F}$ \\
\hline & $\mathrm{C} 18 \mathrm{H} 9 \mathrm{~F} 30 \mathrm{O} 4 \mathrm{P}$ & 2 & & $3,3,4,4,5,5,6,6,7,7,8,8,9,9,10,10,10$ & $\mathrm{C}(\mathrm{COP}(=\mathrm{O})(\mathrm{O}) \mathrm{OCCC}(\mathrm{C}(\mathrm{C}(\mathrm{C}(\mathrm{C}(\mathrm{C}(\mathrm{F})(\mathrm{F}) \mathrm{F})(\mathrm{F}) \mathrm{F})(\mathrm{F}) \mathrm{F})(\mathrm{F}) \mathrm{F})$ \\
\hline & & & & heptadecafluorodecyl $\quad 3,3,4,4,5,5,6,6,7,7,8,8,8$ - & $(\mathrm{F}) \mathrm{F})(\mathrm{F}) \mathrm{F}) \mathrm{C}(\mathrm{C}(\mathrm{C}(\mathrm{C}(\mathrm{C}(\mathrm{C}(\mathrm{C}(\mathrm{C}(\mathrm{F})(\mathrm{F}) \mathrm{F})(\mathrm{F}) \mathrm{F})(\mathrm{F}) \mathrm{F})(\mathrm{F}) \mathrm{F})(\mathrm{F}) \mathrm{F})$ \\
\hline & & & & tridecafluorooctyl hydrogen phosphate & $(\mathrm{F}) \mathrm{F})(\mathrm{F}) \mathrm{F})(\mathrm{F}) \mathrm{F}$ \\
\hline
\end{tabular}




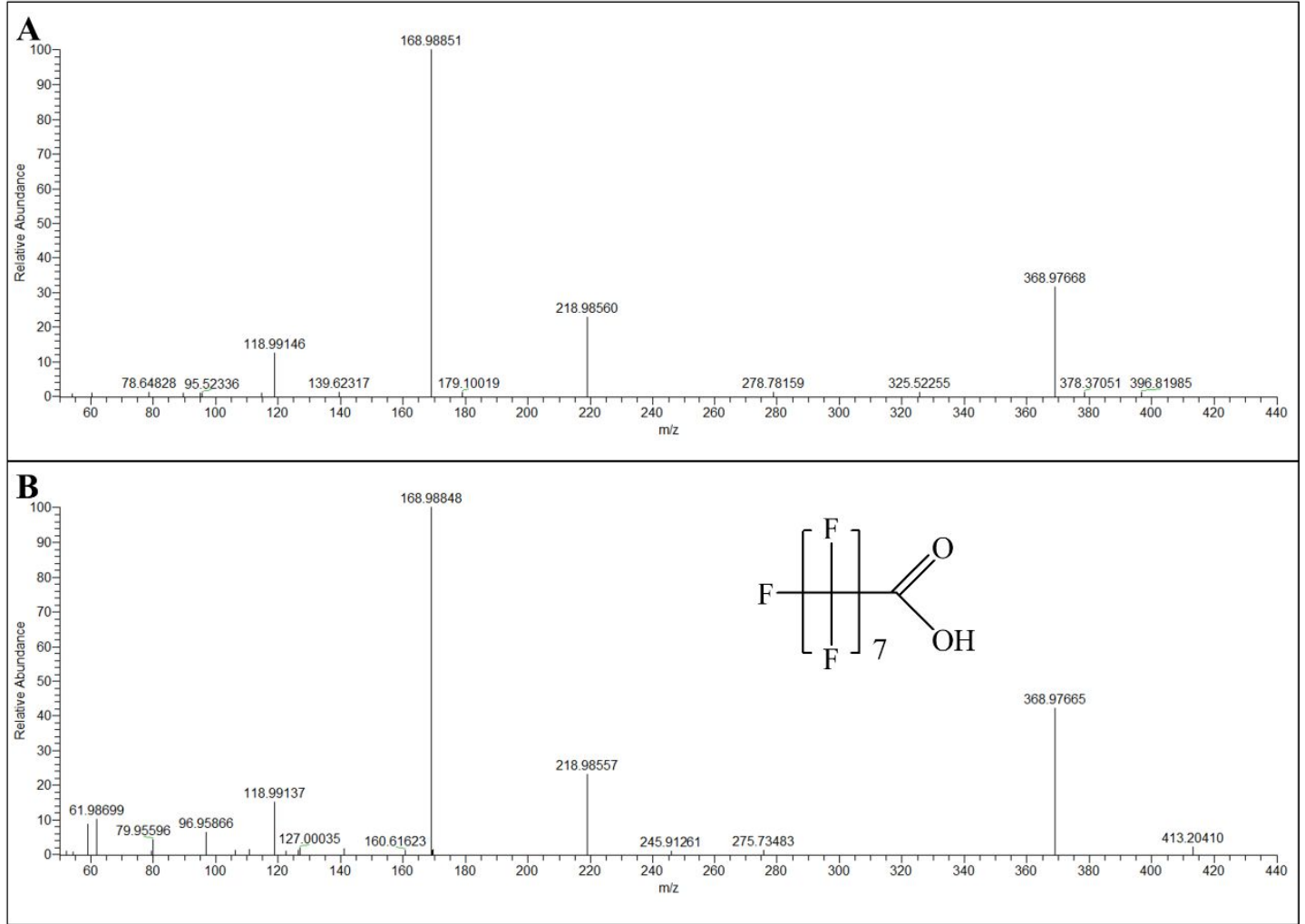

Figure S1. Identification of PFOA (m/z 412.96643). (A) MS/MS spectrum of PFOA in standard. (B) MS/MS spectrum of PFOA in sample. 


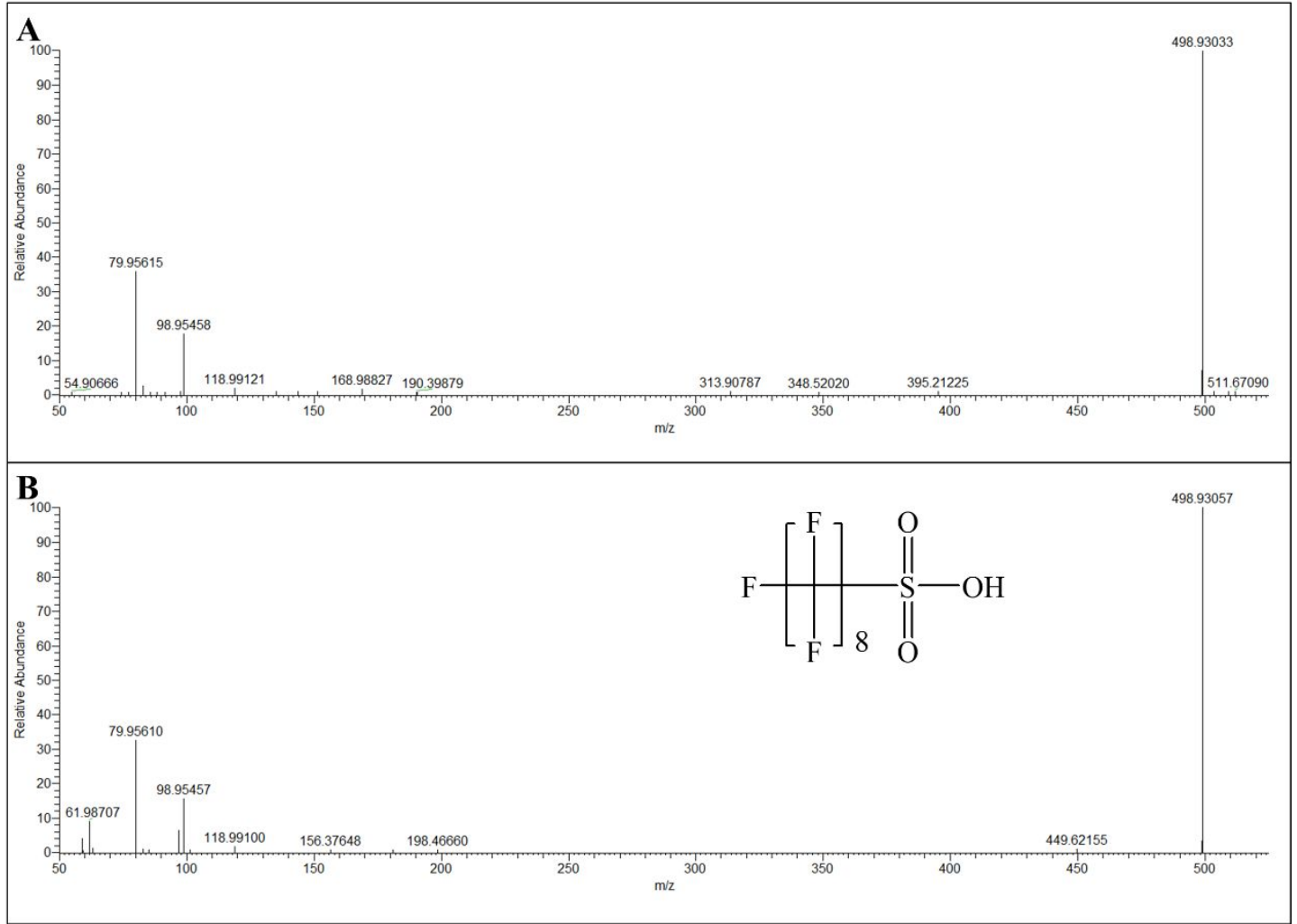

Figure S2. Identification of PFOS (m/z 498.93021). (A) MS/MS spectrum of PFOS in standard. (B) MS/MS spectrum of PFOS in sample. 


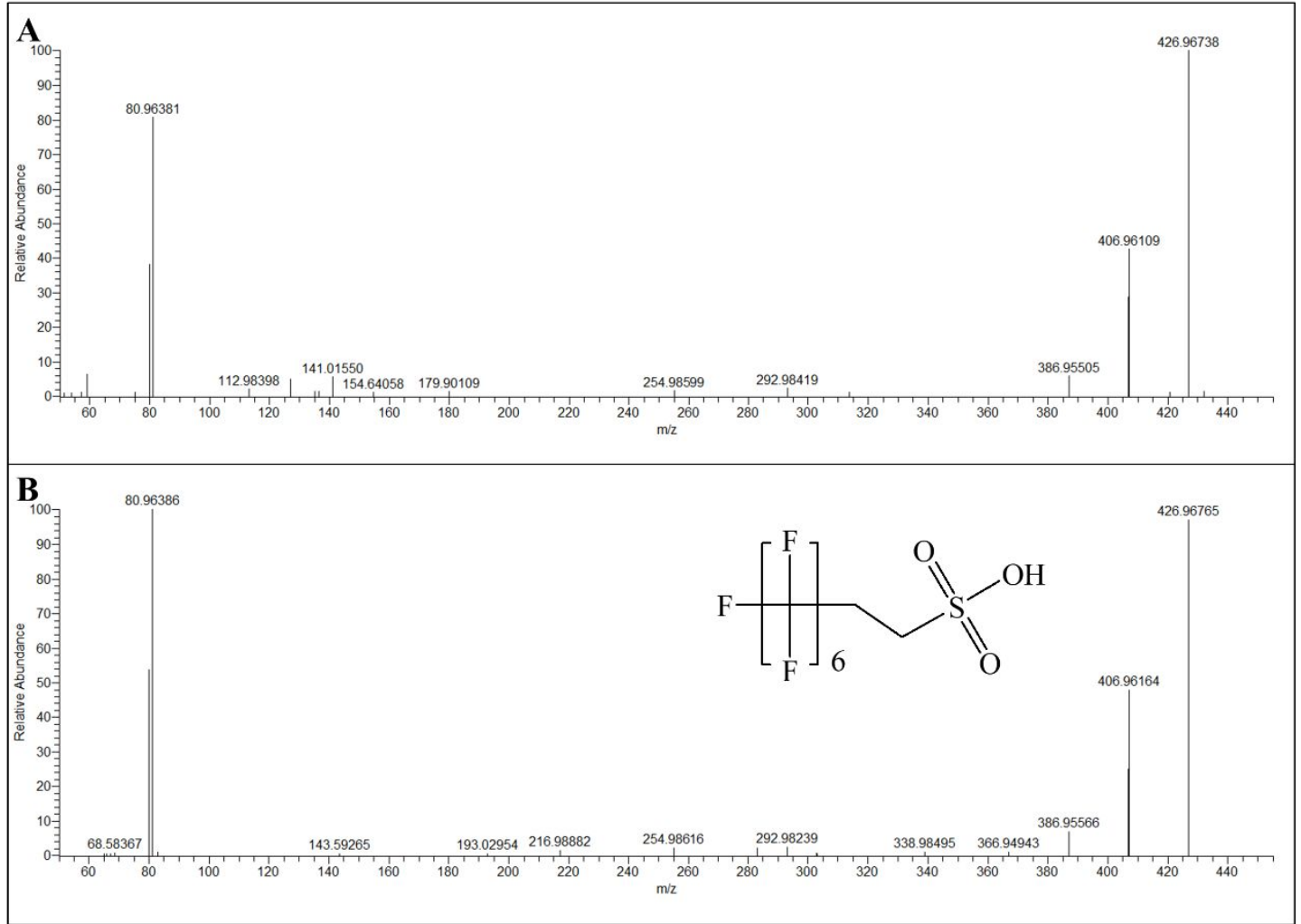

Figure S3. Identification of 6:2 FTS (m/z 426.96791). (A) MS/MS spectrum of 6:2 FTS in standard. (B) MS/MS spectrum of 6:2 FTS in sample. 


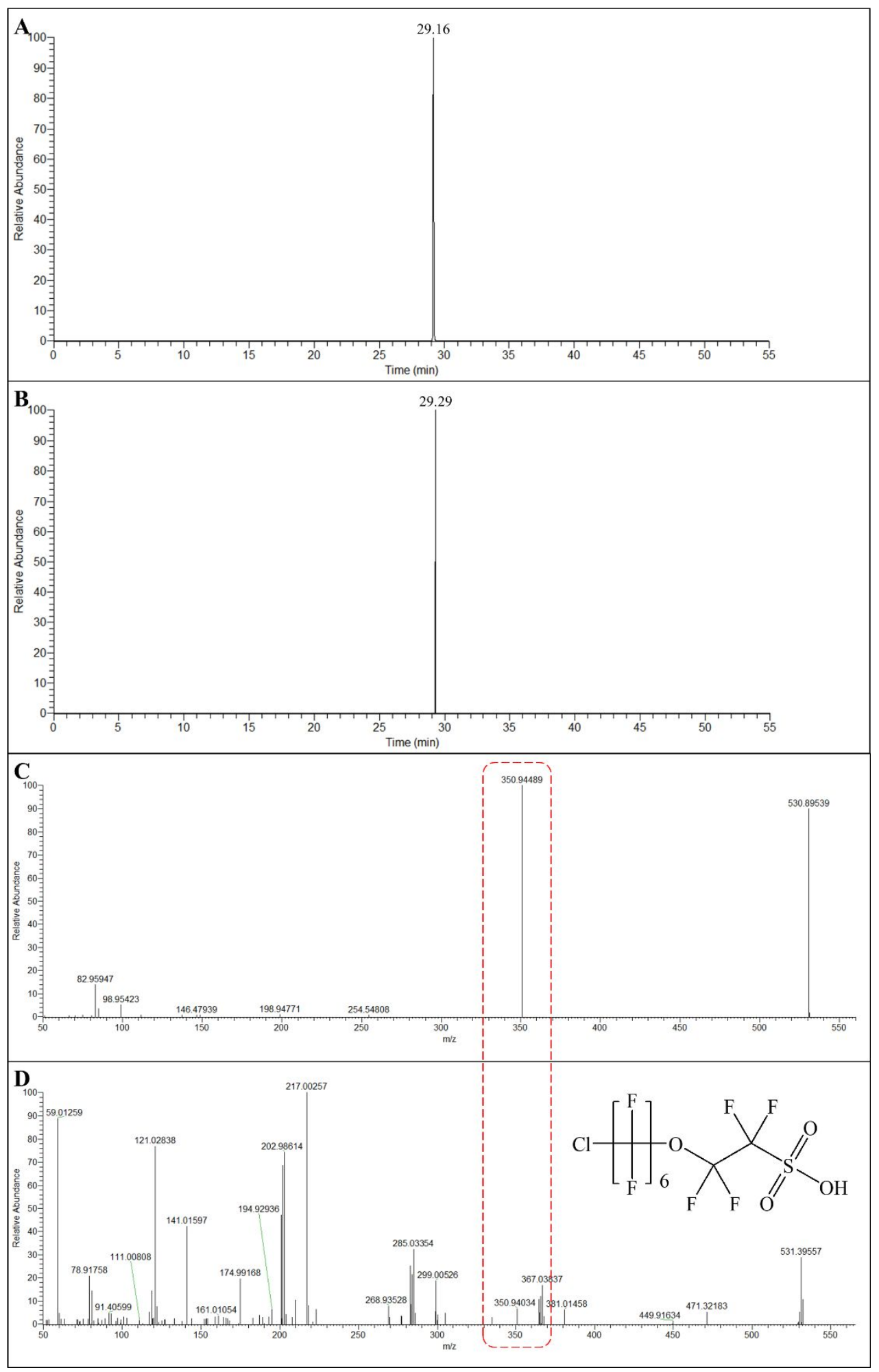

Figure S4. Identification of 6:2 Cl-PFESA (m/z 530.89558). (A) Extract ion chromatogram of 6:2 Cl-PFESA in standard. (B) Extract ion chromatogram of 6:2 Cl- 
PFESA in sample. (C) MS/MS spectrum of 6:2 Cl-PFESA in standard. (D) MS/MS spectrum of 6:2 Cl-PFESA in sample. 


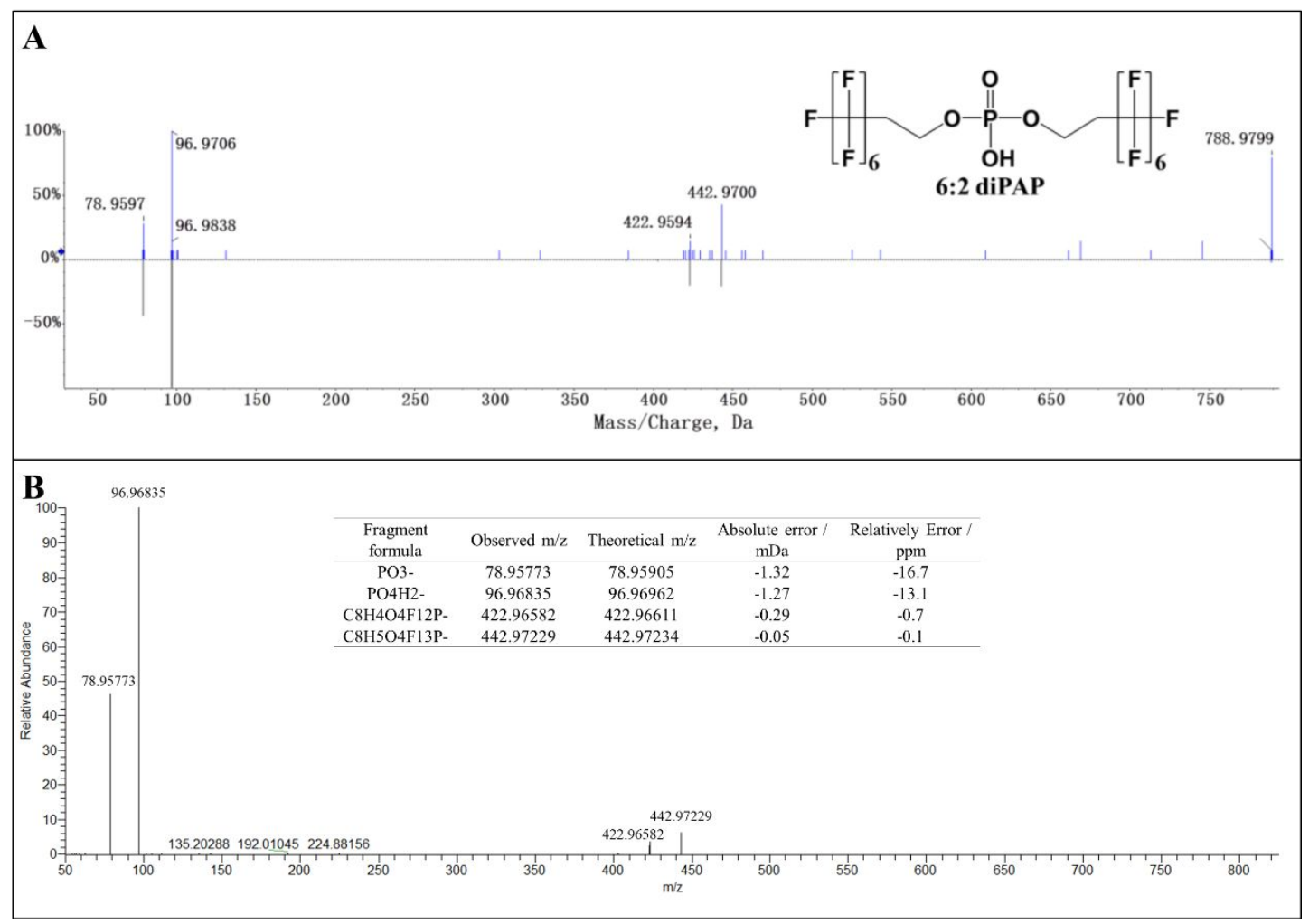

Figure S5. Identification of 6:2 diPAP (m/z 788.97505). (A) MS/MS spectrum of 6:2 diPAP in previous study confirmed by standard. ${ }^{1}$ (B) MS/MS spectrum of 6:2 diPAP in sample. 


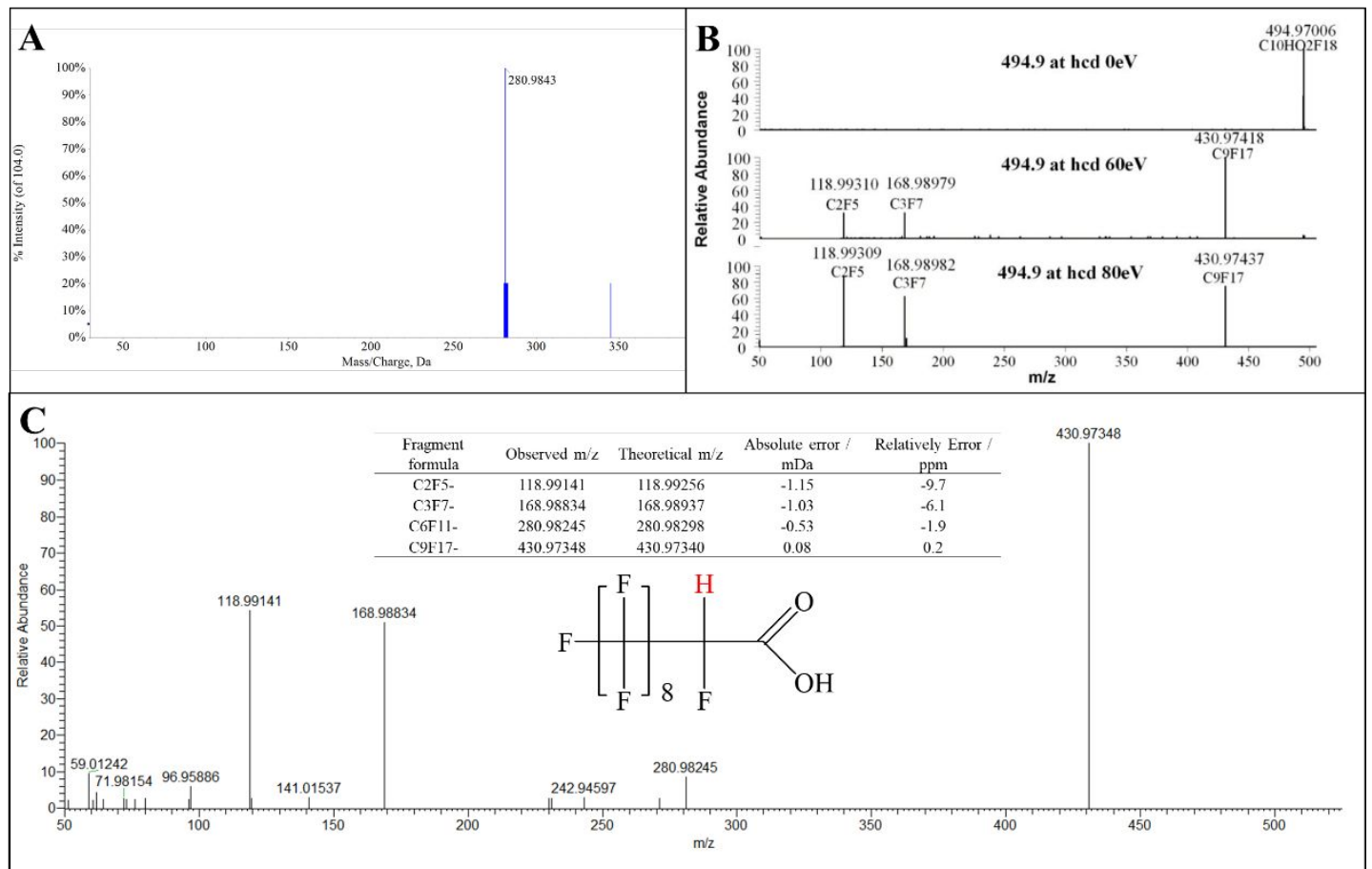

Figure S6. Identification of H-PFCA. (A) MS/MS spectrum of C7 H-PFCA standard (C7H2F12O2, m/z 344.97904). (B) MS/MS spectrum of C10 H-PFCA

$(\mathrm{C} 10 \mathrm{H} 2 \mathrm{~F} 18 \mathrm{O} 2, \mathrm{~m} / \mathrm{z} 494.96946)$ under different energies in previous article. ${ }^{5}(\mathrm{C})$ MS/MS spectrum of C10 H-PFCA in sample. 


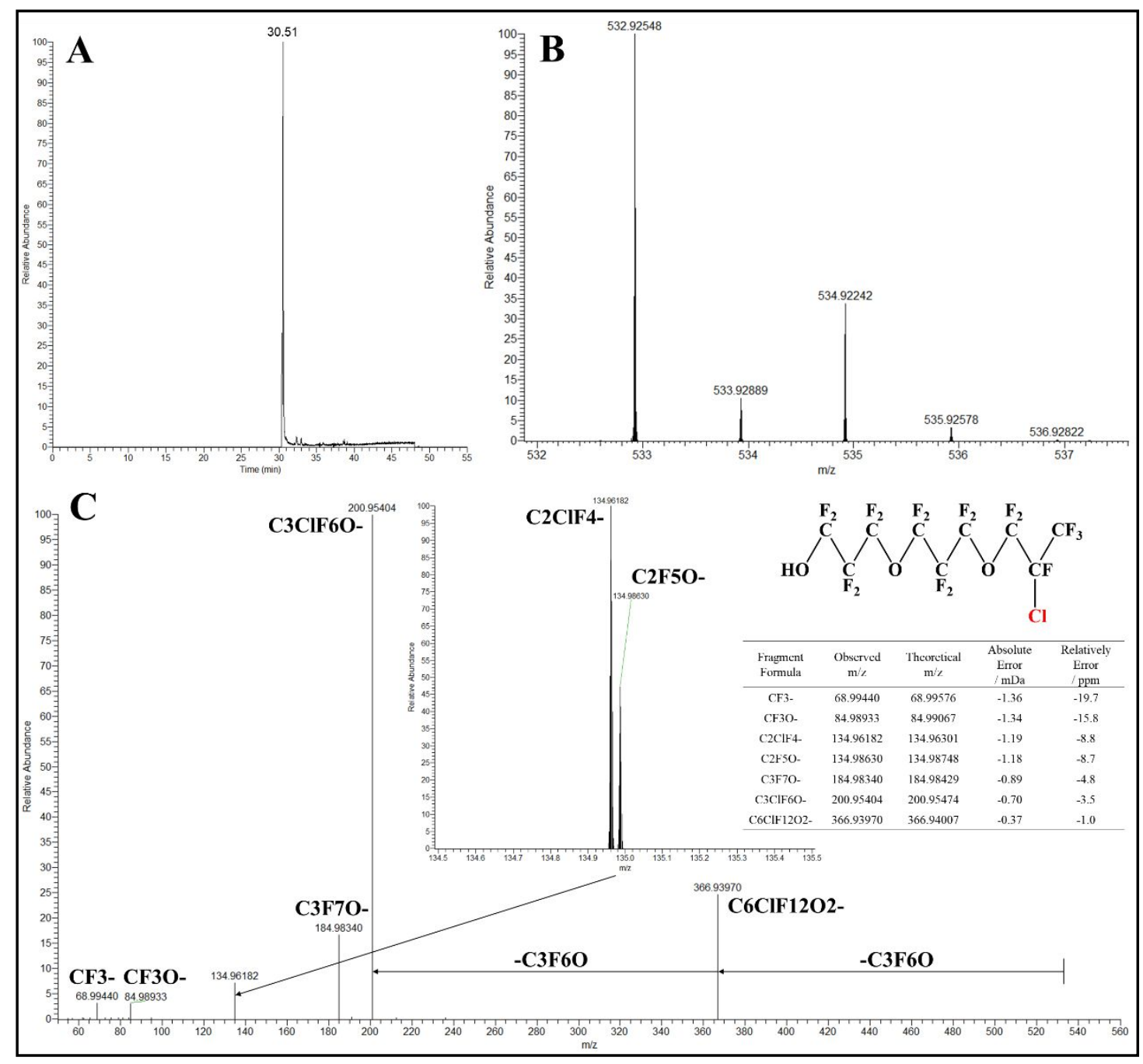

Figure S7. Identification of G03 (m/z 532.92540) in Class 7. (A) Extract ion chromatogram of G03. (B) Isotope distribution of G03 in MS spectrum. (C) MS/MS spectrum of G03 with mass errors for the MS/MS fragments shown in the table. 


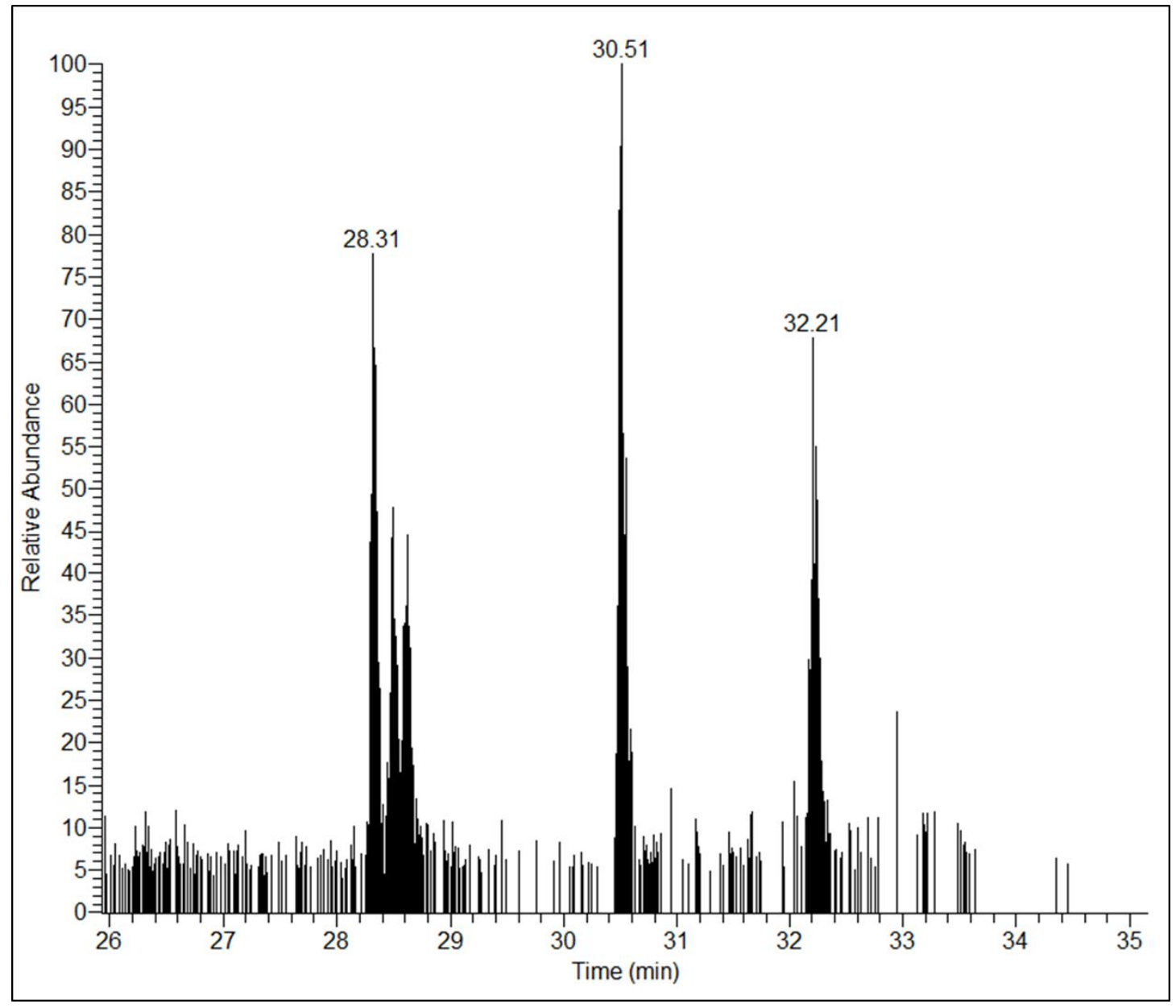

Figure S8. Extract ion chromatogram of G01 (m/z 432.93179) in Class 7. 


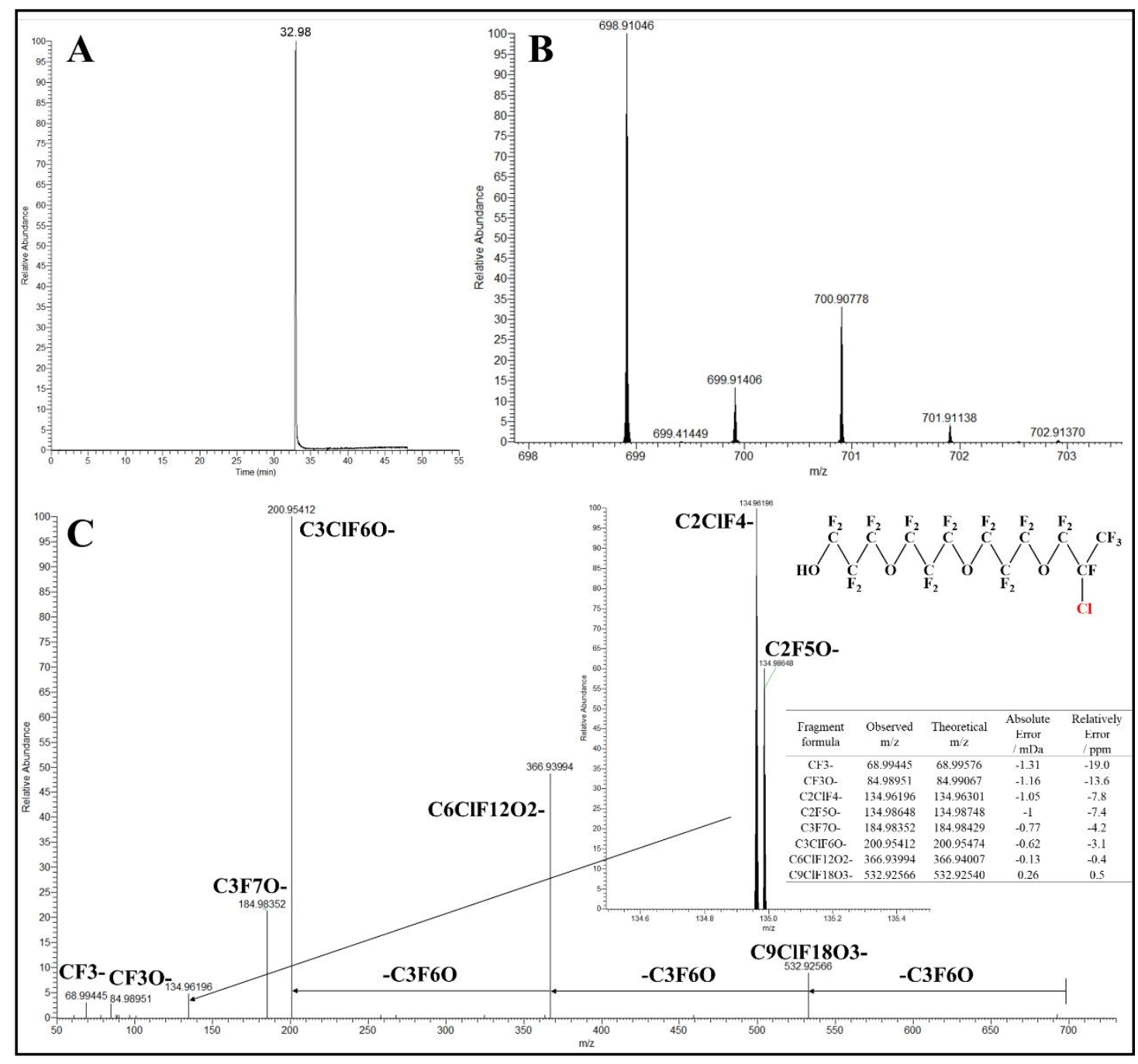

Figure S9. Identification of $\mathrm{H} 04(\mathrm{~m} / \mathrm{z}$ 698.91074) in Class 8. (A) Extract ion chromatogram of H04. (B) Isotope distribution of H04 in MS spectrum. (C) MS/MS spectrum of H04 with mass errors for the MS/MS fragments shown in the table. 


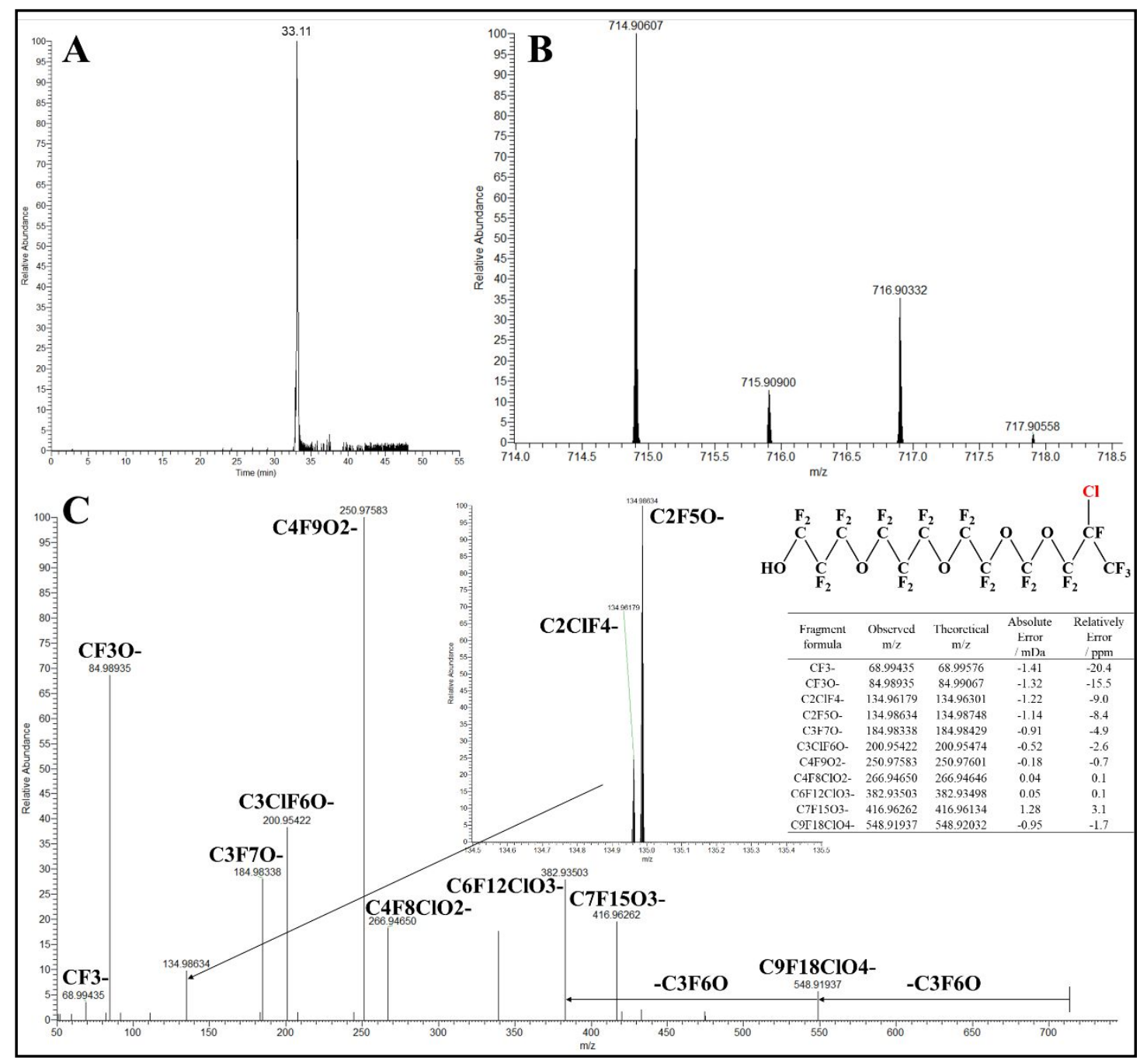

Figure S10. Identification of $\mathrm{I} 02(\mathrm{~m} / \mathrm{z} 714.90565)$ in Class 9. (A) Extract ion chromatogram of I02. (B) Isotope distribution of H04 in MS spectrum. (C) MS/MS spectrum of I02 with mass errors for the MS/MS fragments shown in the table. 


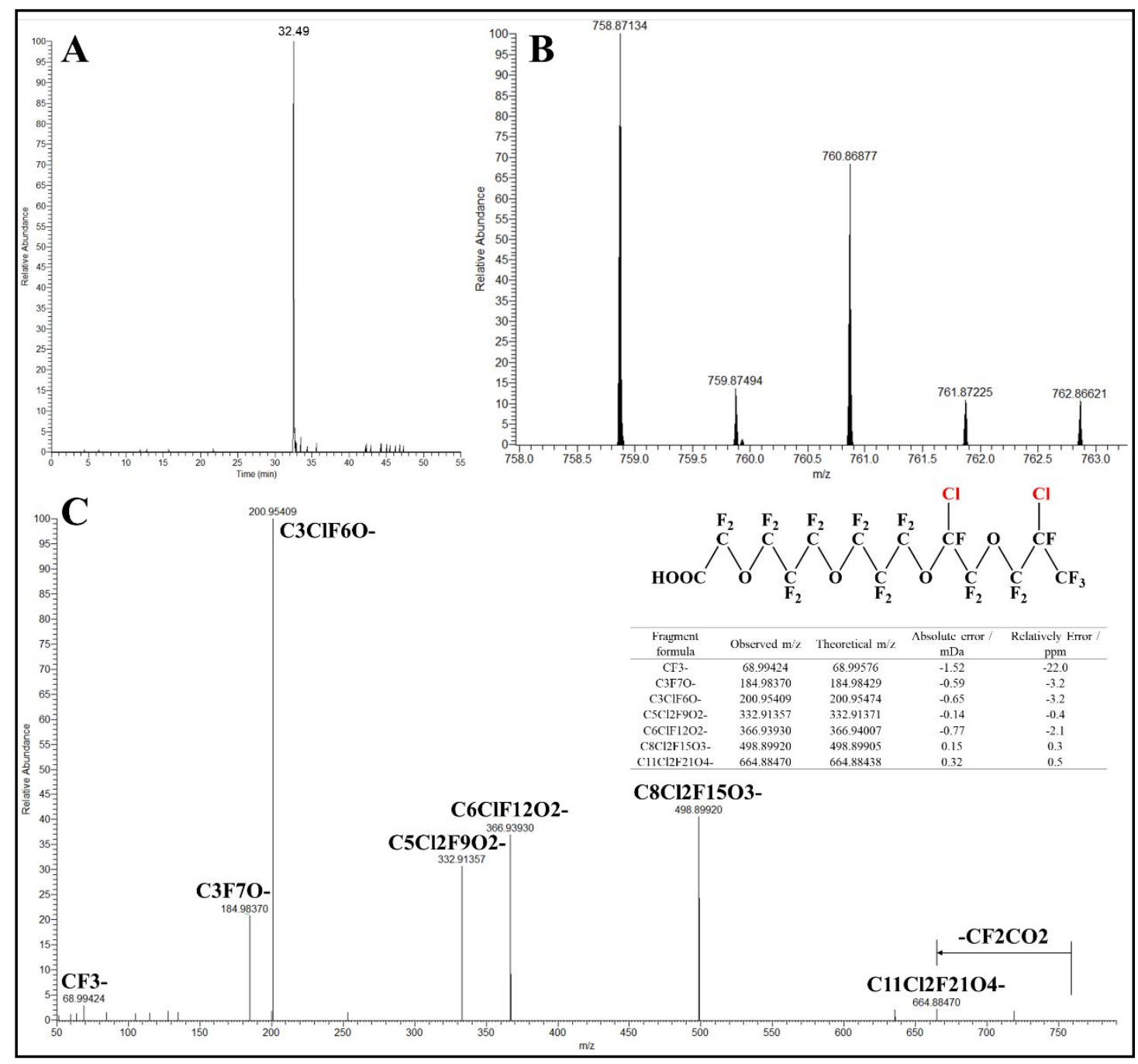

Figure S11. Identification of J02 (m/z 758.87102) in Class 10. (A) Extract ion chromatogram of J02. (B) Isotope distribution of H04 in MS spectrum. (C) MS/MS spectrum of J02 with mass errors for the MS/MS fragments shown in the table. 


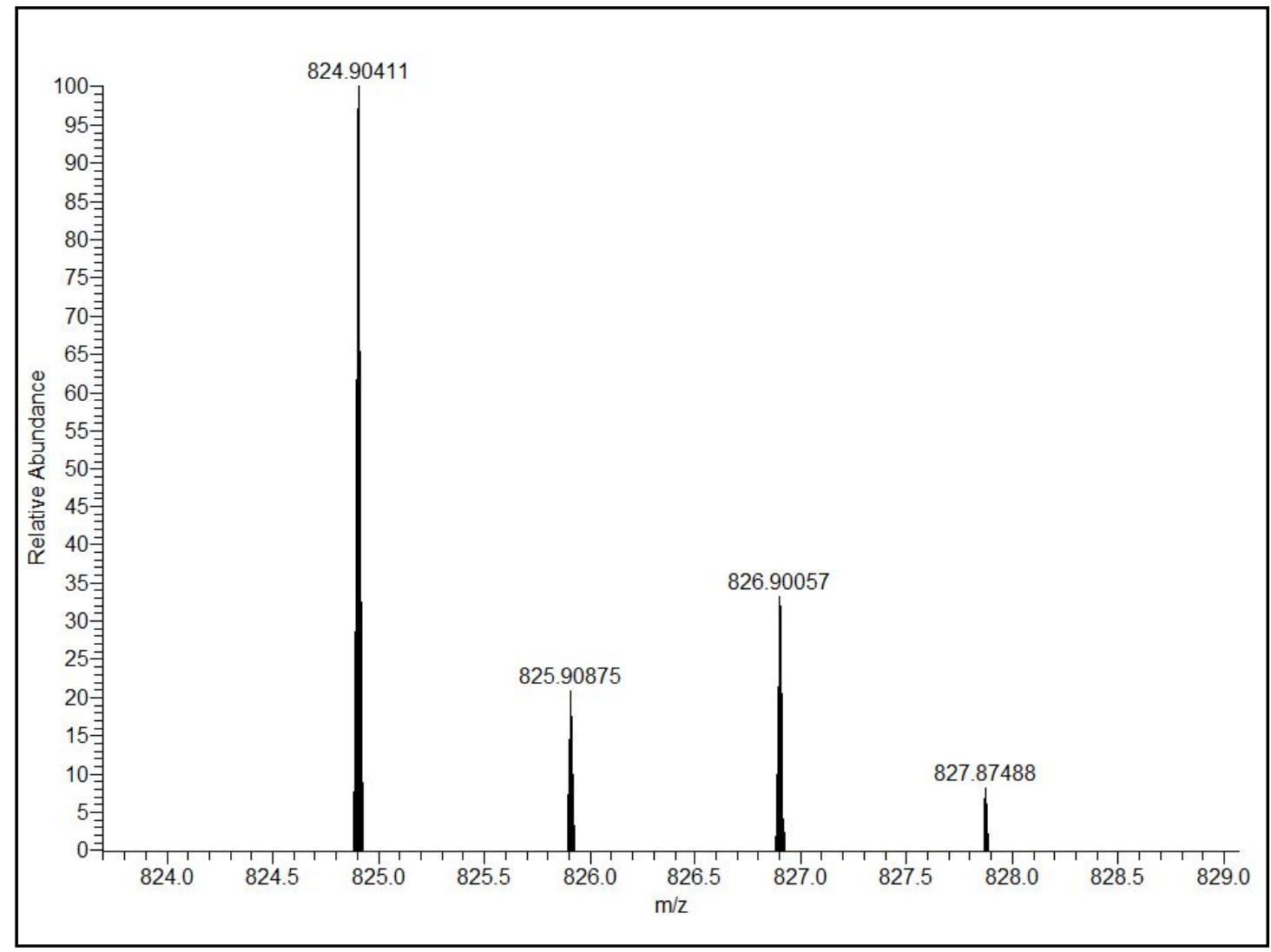

Figure S12. Isotope distribution of K02 (m/z 824.9036) in Class 11. 
Table S7. Potential PFASs homologues found as Level 5. The numbers in the "Particulate Matter" and "Gaseous Phase" show the distribution ratio in each phase and were converted by chromatographic peak area.

\begin{tabular}{|c|c|c|c|c|c|c|c|}
\hline \multirow[b]{2}{*}{ Homologue } & \multirow{2}{*}{$\begin{array}{c}\text { Observe } \\
\text { Molecular } \\
\text { Weight }\end{array}$} & \multirow{2}{*}{$\begin{array}{l}\mathrm{RT} \\
/ \mathrm{min}\end{array}$} & \multicolumn{4}{|c|}{ Particulate Matter } & \multirow{2}{*}{$\begin{array}{c}\text { Gaseous } \\
\text { Phase }\end{array}$} \\
\hline & & & $>10 \mu \mathrm{m}$ & $10-2.5 \mu \mathrm{m}$ & $2.5-1 \mu \mathrm{m}$ & $<1 \mu \mathrm{m}$ & \\
\hline \multirow[t]{3}{*}{ Class 14} & 208.03662 & 6.72 & & 0.21 & 0.49 & 0.30 & 0.002 \\
\hline & 758.00050 & 36.89 & & 0.29 & & 0.29 & 0.42 \\
\hline & 807.99647 & 37.51 & & 0.58 & 0.42 & & \\
\hline \multirow[t]{2}{*}{ Class 15} & 222.08883 & 14.57 & & 0.15 & 0.45 & 0.36 & 0.03 \\
\hline & 372.07811 & 15.40 & & & & & 1 \\
\hline \multirow[t]{3}{*}{ Class 16} & 132.07754 & 3.63 & & 0.13 & & 0.17 & 0.70 \\
\hline & 232.07074 & 11.40 & & & & & 1 \\
\hline & 332.06392 & 33.84 & 0.42 & & 0.19 & & 0.40 \\
\hline \multirow[t]{3}{*}{ Class 17} & 136.09265 & 7.29 & & & & & 1 \\
\hline & 186.08847 & 11.32 & & & & & 1 \\
\hline & 386.07585 & 29.35 & & & & & 1 \\
\hline \multirow[t]{3}{*}{ Class 18} & 198.02702 & 10.82 & 0.30 & 0.20 & 0.19 & 0.30 & 0.002 \\
\hline & 298.01903 & 17.47 & & & & & 1 \\
\hline & 398.01332 & 23.00 & & & & & 1 \\
\hline Class 19 & 284.08726 & 12.50 & & 0.02 & 0.97 & 0.01 & 0.002 \\
\hline \multirow[t]{3}{*}{ Class 20} & 244.13081 & 10.27 & & & & & 1 \\
\hline & 394.12230 & 25.77 & & & & & 1 \\
\hline & 594.10933 & 28.95 & 1 & & & & \\
\hline \multirow[t]{5}{*}{ Class 21} & 238.12007 & 16.55 & & & & & 1 \\
\hline & 288.11317 & 19.14 & & & & & 1 \\
\hline & 388.10713 & 19.36 & & & & & 1 \\
\hline & 488.10011 & 25.77 & & & & & 1 \\
\hline & 588.09968 & 28.22 & & & & & 1 \\
\hline \multirow[t]{3}{*}{ Class 22} & 176.10397 & 6.46 & & 0.05 & 0.23 & 0.15 & 0.57 \\
\hline & 326.09361 & 12.04 & & & & & 1 \\
\hline & 376.09160 & 26.74 & & & & & 1 \\
\hline \multirow[t]{3}{*}{ Class 23} & 128.08260 & 11.34 & & & & & 1 \\
\hline & 278.07249 & 17.04 & & & & & 1 \\
\hline & 378.06840 & 26.01 & & & & & 1 \\
\hline \multirow[t]{3}{*}{ Class 24} & 160.10901 & 7.41 & 0.007 & 0.04 & 0.11 & 0.14 & 0.70 \\
\hline & 310.09868 & 11.77 & & & & & 1 \\
\hline & 410.09385 & 24.05 & & & & & 1 \\
\hline \multirow[t]{3}{*}{ Class 25} & 166.09851 & 17.15 & & & & & 1 \\
\hline & 316.08824 & 18.56 & & & & & 1 \\
\hline & 466.08064 & 18.61 & & & & & 1 \\
\hline
\end{tabular}




\begin{tabular}{|c|c|c|c|c|c|c|c|}
\hline \multirow[b]{2}{*}{ Homologue } & \multirow{2}{*}{$\begin{array}{c}\text { Observe } \\
\text { Molecular } \\
\text { Weight }\end{array}$} & \multirow[b]{2}{*}{$\begin{array}{c}\mathrm{RT} \\
/ \mathrm{min}\end{array}$} & \multicolumn{4}{|c|}{ Particulate Matter } & \multirow{2}{*}{$\begin{array}{c}\text { Gaseous } \\
\text { Phase }\end{array}$} \\
\hline & & & $>10 \mu \mathrm{m}$ & $10-2.5 \mu \mathrm{m}$ & $2.5-1 \mu \mathrm{m}$ & $<1 \mu \mathrm{m}$ & \\
\hline \multirow[t]{2}{*}{ Class 26} & 339.12562 & 14.83 & & & & & 1 \\
\hline & 389.12352 & 18.96 & & & & & 1 \\
\hline \multirow[t]{3}{*}{ Class 27} & 180.07770 & 16.53 & & 0.48 & 0.52 & & \\
\hline & 330.06755 & 22.57 & & & & & 1 \\
\hline & 480.05958 & 23.54 & & & & & 1 \\
\hline \multirow[t]{3}{*}{ Class 28} & 181.10945 & 16.35 & & & & & 1 \\
\hline & 331.09913 & 17.77 & & & & & 1 \\
\hline & 381.09672 & 24.98 & & & & & 1 \\
\hline \multirow[t]{2}{*}{ Class 29} & 343.09901 & 10.89 & & & & & 1 \\
\hline & 443.09422 & 26.90 & & & & & 1 \\
\hline \multirow[t]{3}{*}{ Class 30} & 214.11352 & 19.90 & & & & & 1 \\
\hline & 264.11101 & 20.74 & & & & & 1 \\
\hline & 314.10899 & 25.93 & & & & & 1 \\
\hline \multirow[t]{3}{*}{ Class 31} & 224.06804 & 10.25 & & & & & 1 \\
\hline & 324.06108 & 10.62 & & & & & 1 \\
\hline & 374.06093 & 13.52 & & & & & 1 \\
\hline \multirow[t]{2}{*}{ Class 32} & 242.10892 & 12.40 & & & & & 1 \\
\hline & 342.10390 & 24.88 & & & & & 1 \\
\hline Class 33 & 244.02571 & 2.04 & & 0.73 & & & 0.27 \\
\hline \multirow[t]{3}{*}{ Class 34} & 247.08423 & 13.93 & & & & & 1 \\
\hline & 347.07634 & 20.57 & & & & & 1 \\
\hline & 397.07408 & 34.07 & & & & & 1 \\
\hline \multirow[t]{3}{*}{ Class 35} & 250.12022 & 16.72 & 0.12 & 0.03 & 0.23 & 0.01 & 0.62 \\
\hline & 400.10936 & 19.89 & & & & & 1 \\
\hline & 450.10740 & 31.16 & & & & & 1 \\
\hline \multirow[t]{3}{*}{ Class 36} & 268.13112 & 15.57 & & & & & 1 \\
\hline & 418.12022 & 20.06 & & & & & 1 \\
\hline & 618.11030 & 23.80 & & & & & 1 \\
\hline \multirow[t]{3}{*}{ Class 37} & 295.05156 & 17.20 & & & & & 1 \\
\hline & 345.04939 & 21.74 & & & & & 1 \\
\hline & 395.04323 & 22.59 & & & & & 1 \\
\hline \multirow[t]{3}{*}{ Class 38} & 378.12501 & 23.92 & & & & & 1 \\
\hline & 478.11717 & 24.98 & & & & & 1 \\
\hline & 578.11244 & 31.82 & & & & & 1 \\
\hline
\end{tabular}




\section{References:}

1. Yu, N.; Guo, H.; Yang, J.; Jin, L.; Wang, X.; Shi, W.; Zhang, X.; Yu, H.; Wei, S., Non-Target and Suspect Screening of Per- and Polyfluoroalkyl Substances in Airborne Particulate Matter in China. Environ Sci Technol 2018, 52, (15), 8205-8214.

2. Wang, Y.; Yu, N. Y.; Zhu, X. B.; Guo, H. W.; Jiang, J. G.; Wang, X. B.; Shi, W.;

Wu, J. C.; Yu, H. X.; Wei, S., Suspect and Nontarget Screening of Per- and Polyfluoroalkyl Substances in Wastewater from a Fluorochemical Manufacturing Park. Environ. Sci. Technol. 2018, 52, (19), 11007-11016.

3. NORMAN NETWORK. List of PFAS reported in Non-Target HRMS Studies (Liu et al 2019). http://www.normannetwork.com/sites/default/files/files/suspectListExchange/080419Update/PFASNTRE V19_Liu_etal_17042019.xlsx

4. Schymanski, E. L.; Jeon, J.; Gulde, R.; Fenner, K.; Ruff, M.; Singer, H. P.; Hollender, J., Identifying small molecules via high resolution mass spectrometry: communicating confidence. Environ Sci Technol 2014, 48, (4), 2097-8.

5. Liu, Y. N.; Pereira, A. D.; Martin, J. W., Discovery of C-5-C-17 Poly- and Perfluoroalkyl Substances in Water by In-Line SPE-HPLC-Orbitrap with In-Source Fragmentation Flagging. Anal. Chem. 2015, 87, (8), 4260-4268. 